\title{
Verifiable sufficient conditions for the error bound property of second-order cone complementarity problems
}

\author{
Jane J. Ye* and Jinchuan Zhou ${ }^{\dagger}$
}

March 15, 2018

\begin{abstract}
The error bound property for a solution set defined by a set-valued mapping refers to an inequality that bounds the distance between vectors closed to a solution of the given set by a residual function. The error bound property is a Lipschitz-like/calmness property of the perturbed solution mapping, or equivalently the metric subregularity of the underlining set-valued mapping. It has been proved to be extremely useful in analyzing the convergence of many algorithms for solving optimization problems, as well as serving as a constraint qualification for optimality conditions. In this paper, we study the error bound property for the solution set of a very general second-order cone complementarity problem (SOCCP). We derive some sufficient conditions for error bounds for SOCCP which is verifiable based on the initial problem data.
\end{abstract}

Key words: second-order cone complementarity set, complementarity problem, local error bounds, Lipschitz-like, calmness, metric subregularity, constraint qualifications.

AMS subject classification: 49J53, 90C33.

\section{Introduction}

In this paper we consider a second-order cone complementarity problem (SOCCP) of finding $z \in \mathbb{R}^{n}$ satisfying the second-order cone complementarity system defined as

$$
\begin{aligned}
& \mathcal{K} \ni G(z) \perp H(z) \in \mathcal{K}, \\
& F(z) \in \Lambda,
\end{aligned}
$$

where $\Lambda$ is a closed subset of $\mathbb{R}^{l}, F: \mathbb{R}^{n} \rightarrow \mathbb{R}^{l}, G: \mathbb{R}^{n} \rightarrow \mathbb{R}^{m}, H: \mathbb{R}^{n} \rightarrow \mathbb{R}^{m}$ are continuously differentiable, $a \perp b$ means that vector $a$ is perpendicular to vector $b, \mathcal{K}$ is the Cartesian product of finitely many second-order cones (also called Lorentz cones), i.e.,

$$
\mathcal{K}:=\mathcal{K}_{1} \times \mathcal{K}_{2} \times \cdots \times \mathcal{K}_{J}
$$

${ }^{*}$ Department of Mathematics and Statistics, University of Victoria, Victoria, B.C., Canada V8W 2Y2, e-mail: janeye@uvic.ca. The research of this author was partially supported by NSERC.

${ }^{\dagger}$ Department of Statistics, School of Mathematics and Statistics, Shandong University of Technology, Zibo 255049, P.R. China, e-mail: jinchuanzhou@163.com. This author's work is supported by National Natural Science Foundation of China $(11771255,11101248)$ and Shandong Province Natural Science Foundation (ZR2016AM07). 
with $\mathcal{K}_{i}:=\left\{x=\left(x_{1}, x_{2}\right) \in \mathbb{R} \times \mathbb{R}^{m_{i}-1} \mid x_{1} \geq\left\|x_{2}\right\|\right\}$ being the $m_{i}$-dimensional second-order cone and $m=\sum_{i=1}^{J} m_{i}$.

One of the sources of the second-order cone complementarity system is the KarushKuhn-Tucker (KKT) optimality condition for the second-order cone programming (see e.g. [1, 3, 5]), and the other is the equilibrium system for a Nash game where the constraints involving second-order cones (see e.g. [19]).

Let $\mathcal{F}$ denote the solution set of an SOCCP which contains all $z$ satisfying the secondorder cone complementarity system (11)-(2). In this paper, we study the following error bound property. We say that the second-order cone complementarity system has a local error bound at $z^{*} \in \mathcal{F}$ if there exist a constant $\kappa>0$ and $U$ a neighborhood of $z^{*}$ such that

$$
d(z, \mathcal{F}) \leq \kappa\left\{d_{\Lambda}(F(z))+\sum_{i=1}^{J} d_{\Omega_{i}}\left(G_{i}(z), H_{i}(z)\right)\right\} \quad \forall z \in U,
$$

where $\Omega_{i}:=\left\{(x, y) \mid \mathcal{K}_{i} \ni x \perp y \in \mathcal{K}_{i}\right\}$ is the $m_{i}$-dimensional second-order cone complementarity set. The right hand side of the inequality (3) is a residual function, and hence the existence of a local error bound enables us to use the residual to measure the distance from a point $z$ that is sufficiently close to $z^{*}$ to the solution set $\mathcal{F}$. It is easy to verify that the error bound property at $z^{*}$ is equivalent to the calmness of the set-valued mapping defined by

$$
\mathcal{F}(\alpha, \beta, \gamma):=\left\{\begin{array}{l}
z \mid \begin{array}{l}
\mathcal{K} \ni(G(z)+\alpha) \perp(H(z)+\beta) \in \mathcal{K} \\
F(z)+\gamma \in \Lambda
\end{array}
\end{array}\right\}
$$

at $\left(0,0,0, z^{*}\right) \in$ gph $\mathcal{F}$. Since $\mathcal{F}(0,0,0)=\mathcal{F}$, the solution to the second-order cone complementarity system, the set-valued map $\mathcal{F}(\alpha, \beta, \gamma)$ is the perturbed solution mapping. Hence, the calmness property is a Lipschitz-like property of the perturbed solution mapping: there exist a constant $\kappa>0, U$ a neighborhood of $z^{*}, W$ a neighborhood of $(0,0,0)$ such that

$$
\mathcal{F}(\alpha, \beta, \gamma) \cap U \subseteq \mathcal{F}+\kappa\|(\alpha, \beta, \gamma)\| \bar{B} \quad \forall(\alpha, \beta, \gamma) \in W .
$$

For $i=1, \ldots, J$, it is easy to verify that

$$
(x, y) \in \Omega_{i} \Longleftrightarrow x=\Pi_{\mathcal{K}_{i}}(x-y),
$$

and the following inequality holds:

$$
d_{\Omega_{i}}(x, y) \leq \sqrt{2}\left\|x-\Pi_{\mathcal{K}_{i}}(x-y)\right\|, \quad \forall x, y \in \mathbb{R}^{m_{i}},
$$

where $\Pi_{\mathcal{K}_{i}}(z)$ denotes the metric projection of $z$ onto $\mathcal{K}_{i}$. Therefore, if the error bound property holds with the residual function $d_{\Lambda}(F(z))+\sum_{i=1}^{J} d_{\Omega_{i}}\left(G_{i}(z), H_{i}(z)\right)$, then the error bound property also holds with the natural residual function $d_{\Lambda}(F(z))+\sum_{i=1}^{J} \| G_{i}(z)-$ $\Pi_{\mathcal{K}_{i}}\left(G_{i}(z)-H_{i}(z)\right) \|$.

The error bound property and equivalently the calmness property is a very important property. One of the applications of such a property is the analysis of certain algorithms for solving the second-order cone complementarity problem. In particular, it has recently been discovered that the condition that is crucial to the quadratic convergence of the Newton-type method is not the nonsingularity of the Jacobian per se, but rather one of its consequencesthe error bound property; see [10]. Another application is the constraint qualification for the mathematical program with second-order cone complementarity constraints (SOC-MPCC); see [36]. 
Although the error bound property is an important property, there are very few results on sufficient conditions for the existence of error bounds, and these results are abstract and not easy to verify; see e.g. [9, 24, 26, 31, 32, 33] and references therein. One exception is the case where all mappings $F, G, H$ are affine, $\mathcal{K}$ is polyhedral and $\Lambda$ is the union of finitely many convex polyhedral sets. In this case, the local error bound property holds automatically following from Robinson's result on polyhedral multifunctions [29]. This result, however, depends crucially on the functions $F, G, H$ being affine and the sets $\mathcal{K}, \Lambda$ being polyhedral and the union of finitely many convex polyhedral sets, respectively. The secondorder cone, however, is not polyhedral when the dimension is larger than two, and so even when all the mappings $F, G, H$ are affine and the set $\Lambda$ is the union of finitely many convex polyhedral sets, the local error bound property may not hold without further assumptions if one of the second-order cones $\mathcal{K}_{i}$ has dimension $m_{i} \geq 3$. Another easy to verify case is when the gradient vectors $\nabla G_{i}\left(z^{*}\right)(i=1, \ldots, m), \nabla H_{i}\left(z^{*}\right)(i=1, \ldots, m), \nabla F_{i}\left(z^{*}\right)(i=1, \ldots, l)$ are linearly independent. But this condition is very strong.

The main goal of this paper is to provide a verifiable sufficient condition for the local error bound property for the second-order cone complementarity system (11)-(2). Our condition involves only the first-order and/or the second-order derivatives of the mappings $F, G, H$ at the point of interest, and is therefore efficiently checkable. The basis of our approach is the sufficient conditions for metric subregularity recently developed by Gfrerer [12, 13, 14, Gfrerer and Klatte [15], Gfrerer and Ye [16]. To use these results, we need to compute the tangent cones and the directional normal cones to the second-order cone complementarity set. These results, however, are of independent interest.

We summarize our main contributions as follows:

- We introduce a new concept of inner directional normal cone. A set is said to be directionally regular if the inner directional normal cone coincides with the directional normal cone. It describes the variational geometry of a set along some direction. The directional regularity implies the geometrical derivability. In particular, we show that a convex set is directionally regular. Some useful calculus rules for the directional normal cone are derived.

- We establish exact expressions for the tangent cone and the directional normal cone of the second-order cone complementarity set. Moreover we show that the second-order cone complementarity set, which is nonconvex, is directionally regular, and hence both the tangent cone and the directional normal cone commutes with the Cartesian product of finitely many second-order cone complementarity sets.

- We give sufficient conditions for the existence of error bounds of the second-order cone complementarity problems. These conditions are verifiable based on the initial problem data.

We organize our paper as follows. Section 2 contains the preliminaries. In Section 3, we study certain properties of the directional normal cone introduced by Gfrerer [13] and in Section 4 we derive sufficient conditions for the error bound property of a general system by using directional normal cones. Section 5 is devoted to the formula and the property of the tangent cone to the second-order complementarity set. In Section 6, we derive the exact expressions for the directional normal cone for the second-order cone complementarity set. Finally in Section 7 we present sufficient conditions for error bounds of the second-order cone complementarity system. 
The following notation will be used throughout the paper. We denote by $I$ and $O$ the identity and zero matrix of appropriate dimensions respectively. For a matrix $A$, we denote by $A^{T}$ its transpose. The inner product of two vectors $x, y$ is denoted by $x^{T} y$ or $\langle x, y\rangle$. For any $z \in \mathbb{R}^{n}$, we denote by $\|z\|$ the Euclidean norm. For any nonzero vector $z \in \mathbb{R}^{n}$, the notation $\bar{z}$ stands for the normalized vector $\frac{z}{\|z\|}$. For a function $g: \mathbb{R}^{n} \rightarrow \mathbb{R}$, we denote $g_{+}(z):=\max \{0, g(z)\}$, and if it is vector-valued then the maximum is taken componentwise. For $z=\left(z_{1}, z_{2}\right) \in \mathbb{R} \times \mathbb{R}^{m-1}$, we write its reflection about the $z_{1}$ axis as $\hat{z}:=\left(z_{1},-z_{2}\right)$. Denote by $\mathbb{R} z$ the set $\{t z \mid t \in \mathbb{R}\} . \mathbb{R}_{+} z$ and $\mathbb{R}_{++} z$ where $\mathbb{R}_{+}:=[0, \infty)$ and $\mathbb{R}_{++}:=(0, \infty)$ are similarly defined. For a set $C$, denote by $\operatorname{int} C, \operatorname{cl} C, \operatorname{bd} C, \operatorname{co} C$, $C^{c}$ its interior, closure, boundary, convex hull, and complement, respectively. The polar cone of a set $C$ is $C^{\circ}:=\left\{z \mid z^{T} v \leq 0, \forall v \in C\right\}$ and $v^{\circ}$ is the polar cone of a vector $v$. We denote by $d_{C}(z)$ or $d(z, C)$ the distance from $z$ to $C$. Given a point $z \in \mathbb{R}^{n}$ and $\varepsilon>0$, $B_{\varepsilon}(z)$ denotes an open ball centered at $z$ with radius $\varepsilon$ while $B$ and $\bar{B}$ denote the open and the closed unit ball center at the origin of an appropriate dimension, respectively. For a differentiable mapping $H: \mathbb{R}^{n} \rightarrow \mathbb{R}^{m}$ and a vector $z \in \mathbb{R}^{n}$, we denote by $\nabla H(z)$ the Jacobian matrix of $H$ at $z$. By $o(\cdot)$, we mean that $o(\alpha) / \alpha \rightarrow 0$ as $\alpha \rightarrow 0$. For a set-valued mapping $\Phi: \mathbb{R}^{n} \rightrightarrows \mathbb{R}^{m}$, the graph and domain of $\Phi$ are denoted by $\operatorname{gph} \Phi$ and $\operatorname{dom} \Phi$, respectively, i.e., $\operatorname{gph} \Phi:=\left\{(z, v) \in \mathbb{R}^{n} \times \mathbb{R}^{m} \mid v \in \Phi(z)\right\}$ and $\operatorname{dom} \Phi:=\left\{z \in \mathbb{R}^{n} \mid \Phi(z) \neq \emptyset\right\}$. Finally for any mapping $\varphi: \mathbb{R}^{n} \rightarrow \mathbb{R}^{m}$, we denote the active index set at $z^{*} \in \mathbb{R}^{n}$ by $I_{\varphi}\left(z^{*}\right):=\left\{i \in\{1, \ldots, m\} \mid \varphi_{i}\left(z^{*}\right)=0\right\}$. For simplification of notation, we may write $I_{\varphi}\left(z^{*}\right)$ as $I_{\varphi}$, provided that there is no confusion in the context.

\section{Preliminaries}

In this section, we gather some preliminaries on variational analysis and second-order cone which will be used in paper. Detailed discussions on these subjects can be found in [1, 6, 7, 22, 23, 30] and the papers we refer to.

\subsection{Background in variational analysis}

Let $\Phi: \mathbb{R}^{n} \rightrightarrows \mathbb{R}^{m}$ be a set-valued mapping. We denote by $\lim \sup _{z^{\prime} \rightarrow z} \Phi\left(z^{\prime}\right)$ and $\lim \inf _{z^{\prime} \rightarrow z} \Phi\left(z^{\prime}\right)$ the Painlevé-Kuratowski upper and lower limit, i.e.,

$$
\begin{aligned}
\limsup _{z^{\prime} \rightarrow z} \Phi(z) & :=\left\{v \in \mathbb{R}^{m} \mid \exists z_{k} \rightarrow z, v_{k} \rightarrow v \text { with } v_{k} \in \Phi\left(z_{k}\right) \quad \forall k\right\}, \\
\liminf _{z^{\prime} \rightarrow z} \Phi(z) & :=\left\{v \in \mathbb{R}^{m} \mid \forall z_{k} \rightarrow z, \exists v_{k} \rightarrow v \text { with } v_{k} \in \Phi\left(z_{k}\right) \quad \forall k\right\},
\end{aligned}
$$

respectively.

Let $C \subseteq \mathbb{R}^{n}$ and $z \in C$. The tangent cone of $C$ at $z$ is a closed cone defined by

$$
T_{C}(z):=\limsup _{t \downarrow 0} \frac{C-z}{t}=\left\{u \in \mathbb{R}^{n} \mid \exists t_{k} \downarrow 0, u_{k} \rightarrow u \text { with } z+t_{k} u_{k} \in C \forall k\right\} .
$$

The inner tangent/derivable cone of $C$ at $z$ is defined by

$$
T_{C}^{i}(z):=\liminf _{t \downarrow 0} \frac{C-z}{t}=\left\{u \in \mathbb{R}^{n} \mid \forall t_{k} \downarrow 0, \exists u_{k} \rightarrow u \text { such that } z+t_{k} u_{k} \in C \forall k\right\} .
$$


The regular/Fréchet normal cone of $C$ at $z$ is defined by

$$
\widehat{N}_{C}(z):=\left\{v \in \mathbb{R}^{n} \mid\left\langle v, z^{\prime}-z\right\rangle \leq o\left(\left\|z^{\prime}-z\right\|\right) \forall z^{\prime} \in C\right\} .
$$

The limiting/Mordukhovich normal cone is defined by

$$
N_{C}(z):=\limsup _{z^{\prime} \rightarrow} \widehat{N}_{C}\left(z^{\prime}\right)=\left\{\lim _{i \rightarrow \infty} v_{i} \mid v_{i} \in \widehat{N}_{C}\left(z_{i}\right), \quad z_{i} \stackrel{C}{\rightarrow} z\right\} .
$$

Definition 2.1 We say that a set $C$ is geometrically derivable at a point $z \in C$ if the tangent cone of $C$ coincides with the inner tangent cone of $C$ at $z$, i.e., $T_{C}(z)=T_{C}^{i}(z)$.

Let $\Phi: \mathbb{R}^{n} \rightrightarrows \mathbb{R}^{m}$ be a set-valued mapping and $(x, y) \in \operatorname{gph} \Phi$. The regular coderivative and the limiting/Mordukhovich coderivative of $\Phi$ at $(x, y)$ are the set-valued mappings defined by

$$
\begin{aligned}
& \widehat{D}^{*} \Phi(x, y)(v):=\left\{u \in \mathbb{R}^{n} \mid(u,-v) \in \widehat{N}_{\mathrm{gph} \Phi}(x, y)\right\}, \\
& D^{*} \Phi(x, y)(v):=\left\{u \in \mathbb{R}^{n} \mid(u,-v) \in N_{\mathrm{gph} \Phi}(x, y)\right\},
\end{aligned}
$$

respectively. We omit $y$ in the coderivative notations if the set-valued map $\Phi$ is single-valued at $x$.

For a single-valued mapping $\Phi: \mathbb{R}^{n} \rightarrow \mathbb{R}^{m}$, the $\mathrm{B}$ (ouligand)-subdifferential $\partial_{B} \Phi$ is defined as

$$
\partial_{B} \Phi(z)=\left\{\lim _{k \rightarrow \infty} \nabla \Phi\left(z_{k}\right) \mid z_{k} \rightarrow z, \Phi \text { is differentiable at } z_{k}\right\} .
$$

If $\Phi$ is a continuously differentiable single-valued map, then

$$
\widehat{D}^{*} \Phi(z)=D^{*} \Phi(z)=\left\{\nabla \Phi(z)^{T}\right\} .
$$

\subsection{Background in variational analysis associated with the second-order cone}

Let $\mathcal{K}$ be the $m$-dimensional second-order cone. The topological interior and the boundary of $\mathcal{K}$ are

$$
\operatorname{int} \mathcal{K}=\left\{\left(x_{1}, x_{2}\right) \in \mathbb{R} \times \mathbb{R}^{m-1} \mid x_{1}>\left\|x_{2}\right\|\right\}, \quad \operatorname{bd} \mathcal{K}=\left\{\left(x_{1}, x_{2}\right) \in \mathbb{R} \times \mathbb{R}^{m-1} \mid x_{1}=\left\|x_{2}\right\|\right\},
$$

respectively.

Proposition 2.1 (see e.g. [36, Proposition 2.2]) For any $x, y \in b d \mathcal{K} \backslash\{0\}$, the following equivalence holds:

$$
x^{T} y=0 \Longleftrightarrow y=k \hat{x} \text { with } k=y_{1} / x_{1}>0 \Longleftrightarrow y=k \hat{x} \text { with } k \in \mathbb{R}_{++} .
$$

For any given nonzero vector $z:=\left(z_{1}, z_{2}\right) \in \mathbb{R} \times \mathbb{R}^{m-1}$, we denote by

$$
c_{1}(z)=\frac{1}{2}\left(1,-\bar{z}_{2}\right), \quad c_{2}(z)=\frac{1}{2}\left(1, \bar{z}_{2}\right)
$$

the spectral vectors of $z$, where $\bar{z}_{2}$ is any vector $w \in \mathbb{R}^{m-1}$ with $\|w\|=1$ if $z_{2}=0$. For $z \in \mathbb{R}^{m}$, let $\Pi_{\mathcal{K}}(z)$ be the metric projection of $z$ onto $\mathcal{K}$ and $\Pi_{\mathcal{K}}^{\prime}(z ; h)$ the directional derivative of $\Pi_{\mathcal{K}}$ at $z$ in direction $h$. The following proposition summarizes its formula (see [25. Lemma 2]). 
Proposition 2.2 Let $\mathcal{K}$ be the m-dimensional second-order cone. The mapping $\Pi_{\mathcal{K}}(\cdot)$ is directionally differentiable at any $z \in \mathbb{R}^{m}$ and for any $h \in \mathbb{R}^{m}$,

(i) if $z \in$ int $\mathcal{K}$ or $z \in-$ int $\mathcal{K}$ or $z \in(-\mathcal{K} \cup \mathcal{K})^{c}$, then $\Pi_{\mathcal{K}}^{\prime}(z ; h)=\nabla \Pi_{\mathcal{K}}(z) h$;

(ii) if $z \in b d \mathcal{K} \backslash\{0\}$, then $\Pi_{\mathcal{K}}^{\prime}(z ; h)=h-2\left(c_{1}(z)^{T} h\right)_{-} c_{1}(z)$;

(iii) if $z \in-b d \mathcal{K} \backslash\{0\}$, then $\Pi_{\mathcal{K}}^{\prime}(z ; h)=2\left(c_{2}(z)^{T} h\right)_{+} c_{2}(z)$;

(iv) if $z=0$, then $\Pi_{\mathcal{K}}^{\prime}(z ; h)=\Pi_{\mathcal{K}}(h)$.

The following proposition summarizes the regular and the limiting coderivatives of the metric projection operator (see [25, Lemma 1 and Theorems 1 and 2]).

Proposition 2.3 Let $\mathcal{K}$ be the $m$-dimensional second-order cone.

(i) If $z \in$ int $\mathcal{K}$, then $\Pi_{\mathcal{K}}$ is differentiable and $\nabla \Pi_{\mathcal{K}}(z)=I$.

(ii) If $z \in-$ int $\mathcal{K}$, then $\Pi_{\mathcal{K}}$ is differentiable and $\nabla \Pi_{\mathcal{K}}(z)=\{O\}$.

(iii) If $z \in(-\mathcal{K} \cup \mathcal{K})^{c}$, then $\Pi_{\mathcal{K}}$ is differentiable and

$$
\nabla \Pi_{\mathcal{K}}(z)=\frac{1}{2}\left(1+\frac{z_{1}}{\left\|z_{2}\right\|}\right) I+\frac{1}{2}\left[\begin{array}{cc}
-\frac{z_{1}}{\left\|z_{2}\right\|} & \bar{z}_{2}^{T} \\
\bar{z}_{2} & -\frac{z_{1}}{\left\|z_{2}\right\|} \bar{z}_{2} \bar{z}_{2}^{T}
\end{array}\right] .
$$

(iv) If $z \in b d \mathcal{K} \backslash\{0\}$, then

$$
\begin{aligned}
\widehat{D}^{*} \Pi_{\mathcal{K}}(z)\left(u^{*}\right) & =\left\{x^{*} \mid u^{*}-x^{*} \in \mathbb{R}_{+} c_{1}(z),\left\langle x^{*}, c_{1}(z)\right\rangle \geq 0\right\}, \\
D^{*} \Pi_{\mathcal{K}}(z)\left(u^{*}\right) & =\partial_{B} \Pi_{\mathcal{K}}(z) u^{*} \cup\left\{x^{*} \mid u^{*}-x^{*} \in \mathbb{R}_{+} c_{1}(z),\left\langle x^{*}, c_{1}(z)\right\rangle \geq 0\right\},
\end{aligned}
$$

and

$$
\partial_{B} \Pi_{\mathcal{K}}(z)=\left\{I, I+\frac{1}{2}\left[\begin{array}{cc}
-1 & \bar{z}_{2}^{T} \\
\bar{z}_{2} & -\bar{z}_{2} \bar{z}_{2}^{T}
\end{array}\right]\right\}
$$

(v) If $z \in-b d \mathcal{K} \backslash\{0\}$, then

$$
\begin{aligned}
& \widehat{D}^{*} \Pi_{\mathcal{K}}(z)\left(u^{*}\right)=\left\{x^{*} \mid x^{*} \in \mathbb{R}_{+} c_{2}(z),\left\langle u^{*}-x^{*}, c_{2}(z)\right\rangle \geq 0\right\}, \\
& D^{*} \Pi_{\mathcal{K}}(z)\left(u^{*}\right)=\partial_{B} \Pi_{\mathcal{K}}(z) u^{*} \cup\left\{x^{*} \mid x^{*} \in \mathbb{R}_{+} c_{2}(z),\left\langle u^{*}-x^{*}, c_{2}(z)\right\rangle \geq 0\right\},
\end{aligned}
$$

and

$$
\partial_{B} \Pi_{\mathcal{K}}(z)=\left\{O, \frac{1}{2}\left[\begin{array}{cc}
1 & \bar{z}_{2}^{T} \\
\bar{z}_{2} & \bar{z}_{2} \bar{z}_{2}^{T}
\end{array}\right]\right\} .
$$

(vi) If $z=0$, then

$$
\begin{aligned}
\widehat{D}^{*} \Pi_{\mathcal{K}}(z)\left(u^{*}\right)= & \left\{x^{*} \mid x^{*} \in \mathcal{K}, u^{*}-x^{*} \in \mathcal{K}\right\} . \\
D^{*} \Pi_{\mathcal{K}}(z)\left(u^{*}\right)= & \partial_{B} \Pi_{\mathcal{K}}(0) u^{*} \cup\left\{x^{*} \mid x^{*} \in \mathcal{K}, u^{*}-x^{*} \in \mathcal{K}\right\} \\
& \cup \bigcup_{\xi \in C}\left\{x^{*} \mid u^{*}-x^{*} \in \mathbb{R}_{+} \xi,\left\langle x^{*}, \xi\right\rangle \geq 0\right\} \\
& \cup \bigcup_{\eta \in C}\left\{x^{*} \mid x^{*} \in \mathbb{R}_{+} \eta,\left\langle u^{*}-x^{*}, \eta\right\rangle \geq 0\right\},
\end{aligned}
$$


where $C:=\left\{\frac{1}{2}(1, w) \mid w \in \mathbb{R}^{m-1},\|w\|=1\right\}$ and

$$
\partial_{B} \Pi_{\mathcal{K}}(0)=\{O, I\} \cup\left\{\frac{1}{2}\left[\begin{array}{cc}
1 & w^{T} \\
w & 2 \alpha I+(1-2 \alpha) w w^{T}
\end{array}\right] \mid w \in \mathbb{R}^{m-1}, \quad\|w\|=1, \quad \alpha \in[0,1]\right\} .
$$

Proposition 2.4 [37, Proposition 2.1] Let $(x, y) \in \Omega:=\left\{(x, y) \mid x \in \mathcal{K}, y \in \mathcal{K}, x^{T} y=0\right\}$. Then

$$
\begin{aligned}
& \widehat{N}_{\Omega}(x, y)=\left\{(u, v) \mid-v \in \widehat{D}^{*} \Pi_{\mathcal{K}}(x-y)(-u-v)\right\} \\
& N_{\Omega}(x, y)=\left\{(u, v) \mid-v \in D^{*} \Pi_{\mathcal{K}}(x-y)(-u-v)\right\} .
\end{aligned}
$$

The exact formula of the regular normal cone and limiting normal cone of $\Omega$ have been established in [37.

Proposition 2.5 [37, Theorem 3.1] Let $(x, y)$ be in the m-dimensional second-order cone complementarity set $\Omega$. Then

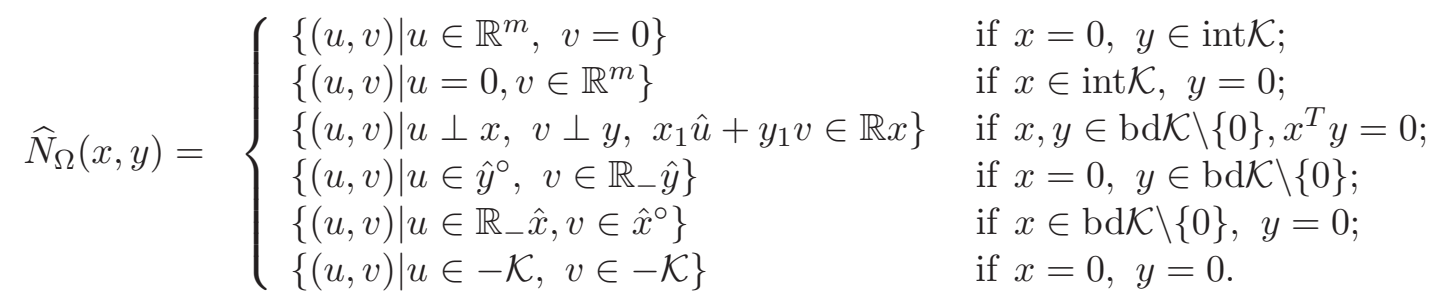

Proposition 2.6 37, Theorem 3.3] Let $(x, y)$ be in the $m$-dimensional second-order cone complementarity set $\Omega$. Then

$N_{\Omega}(x, y)=\widehat{N}_{\Omega}(x, y)= \begin{cases}\left\{(u, v) \mid u \in \mathbb{R}^{m}, v=0\right\} & \text { if } x=0, y \in \operatorname{int} \mathcal{K} ; \\ \left\{(u, v) \mid u=0, v \in \mathbb{R}^{m}\right\} & \text { if } x \in \operatorname{int} \mathcal{K}, y=0 ; \\ \left\{(u, v) \mid u \perp x, v \perp y, x_{1} \hat{u}+y_{1} v \in \mathbb{R} x\right\} & \text { if } x, y \in \operatorname{bd} \mathcal{K} \backslash\{0\} .\end{cases}$

For $x=0, y \in \mathrm{bd} \mathcal{K} \backslash\{0\}$,

$$
N_{\Omega}(x, y)=\left\{(u, v) \mid u \in \mathbb{R}^{m}, v=0 \quad \text { or } \quad u \perp \hat{y}, v \in \mathbb{R} \hat{y} \quad \text { or }\langle u, \hat{y}\rangle \leq 0, v \in \mathbb{R}_{-} \hat{y}\right\}
$$

for $x \in \mathrm{bd} \mathcal{K} \backslash\{0\}, y=0$,

$$
N_{\Omega}(x, y)=\left\{(u, v) \mid u=0, v \in \mathbb{R}^{m} \text { or } u \in \mathbb{R} \hat{x}, v \perp \hat{x} \quad \text { or } u \in \mathbb{R}_{-} \hat{x},\langle v, \hat{x}\rangle \leq 0\right\} ;
$$

for $x=y=0$,

$$
\begin{aligned}
N_{\Omega}(x, y)= & \left\{(u, v) \mid u \in-\mathcal{K}, v \in-\mathcal{K} \text { or } u \in \mathbb{R}^{m}, v=0 \text { or } u=0, v \in \mathbb{R}^{m}\right. \\
& \text { or } u \in \mathbb{R}_{-} \xi, v \in \xi^{\circ} \text { or } u \in \xi^{\circ}, v \in \mathbb{R}_{-} \xi \\
& \text { or } u \perp \xi, v \perp \hat{\xi}, \alpha \hat{u}+(1-\alpha) v \in \mathbb{R} \xi, \text { for some } \alpha \in[0,1], \xi \in C\}
\end{aligned}
$$

where

$$
C:=\left\{(1, w) \mid w \in \mathbb{R}^{m-1},\|w\|=1\right\}
$$




\section{Calculus for directional normal cones}

Recently a directional version of the limiting normal cone was introduced by Gfrerer [13] and used to derive sufficient conditions for metric subregularity, which form the basis for our approach. Since calculus for the directional normal cone is very important and the existing results are rather rare, in this section, we develop some calculus for the directional normal cone. First, we recall the definition of a directional normal cone.

Definition 3.1 Given a set $C \subseteq \mathbb{R}^{n}$, a point $z \in C$ and a direction $d \in \mathbb{R}^{n}$, the limiting normal cone to $C$ in direction $d$ at $z$ is defined by

$N_{C}(z ; d):=\limsup _{\substack{t \downarrow 0 \\ d^{\prime} \rightarrow d}} \widehat{N}_{C}\left(z+t d^{\prime}\right)=\left\{v \mid \exists t_{k} \downarrow 0, d_{k} \rightarrow d, v_{k} \rightarrow v\right.$ with $\left.v_{k} \in \widehat{N}_{C}\left(z+t_{k} d_{k}\right), \forall k\right\}$.

We define the concept of the inner directional normal cone as follows.

Definition 3.2 Given a set $C \subseteq \mathbb{R}^{n}$, a point $z \in C$ and a direction $d \in \mathbb{R}^{n}$, the inner limiting normal cone to $C$ in direction $d$ at $z$ is defined by

$$
N_{C}^{i}(z ; d):=\left\{v \mid \forall t_{k} \rightarrow 0, \exists d_{k} \rightarrow d, v_{k} \rightarrow v \text { with } v_{k} \in \widehat{N}_{C}\left(z+t_{k} d_{k}\right), \forall k\right\} .
$$

The following results follow from definition immediately.

Proposition 3.1 For any set $C$ and any $z \in C$,

$$
\operatorname{dom} N_{C}(z ; \cdot)=T_{C}(z), \quad \operatorname{dom} N_{C}^{i}(z ; \cdot)=T_{C}^{i}(z) .
$$

It is easy to see that $N_{C}^{i}(z ; d) \subseteq N_{C}(z ; d) \subseteq N_{C}(z)$ for any $d$ and $N_{C}(z ; 0)=N_{C}(z)$.

Definition 3.3 Given a subset $C$ in $\mathbb{R}^{n}$ and $z \in C, d \in \mathbb{R}^{n}$, we say that the set $C$ is regular at $z$ in direction $d$ if

$$
N_{C}(z ; d)=N_{C}^{i}(z ; d) .
$$

If the above formula holds for all $d$, we say that $C$ is directionally regular at $z$. If $C$ is directionally regular at any point $z \in C$, then we say that $C$ is directionally regular.

It is clear that set $C$ is regular in direction $d$ for any $d \notin T_{C}(z)$, since both sides of (5) are empty in this case. It follows from Proposition 3.1 that the directional regularity implies the geometric derivability.

Corollary 3.1 If the set $C$ is directionally regular at $z \in C$, then $C$ is geometrically derivable at $z \in C$.

An important property of the limiting normal cone is that it commutes with the Cartesian product (see e.g. [23, Proposition 1.2]): for any sets $A_{1}, \ldots, A_{I}$,

$$
N_{A_{1} \times \cdots \times A_{I}}\left(z_{1}, \ldots, z_{I}\right)=N_{A_{1}}\left(z_{1}\right) \times \cdots \times N_{A_{I}}\left(z_{I}\right) .
$$

It is easy to verify that this property holds for the inner directional normal cones, i.e.,

$$
N_{A_{1} \times \cdots \times A_{I}}^{i}\left(\left(z_{1}, \ldots, z_{I}\right) ;\left(d_{1}, \ldots, d_{I}\right)\right)=N_{A_{1}}^{i}\left(z_{1} ; d_{1}\right) \times \cdots \times N_{A_{I}}^{i}\left(z_{I} ; d_{I}\right) .
$$

For directional normal cones, this kind of property does not come free. Fortunately, it holds under the directional regularity. 
Proposition 3.2 The inclusion

$$
N_{A \times B}((x, y) ;(d, w)) \subseteq N_{A}(x ; d) \times N_{B}(y ; w)
$$

holds for any given sets $A \subseteq \mathbb{R}^{n}, B \subseteq \mathbb{R}^{m}$, any point $(x, y) \in A \times B$, and any direction $(d, w) \in \mathbb{R}^{n} \times \mathbb{R}^{m}$. Moreover if either $A$ is regular at $x$ in direction $d$ or $B$ is regular at $y$ in direction $w$, then

$$
N_{A \times B}((x, y) ;(d, w))=N_{A}(x ; d) \times N_{B}(y ; w) .
$$

If $A$ and $B$ are regular in directions $d, w$ respectively, then $A \times B$ is regular in direction $(d, w)$.

Proof. Note that

$$
\begin{aligned}
N_{A \times B}((x, y) ;(d, w)) & =\limsup _{\substack{t \downarrow 0 \\
\left(d^{\prime}, w^{\prime}\right) \rightarrow(d, w)}} \widehat{N}_{A \times B}\left((x, y)+t\left(d^{\prime}, w^{\prime}\right)\right) \\
& =\limsup _{\substack{t \downarrow 0 \\
\left(d^{\prime}, w^{\prime}\right) \rightarrow(d, w)}} \widehat{N}_{A}\left(x+t d^{\prime}\right) \times \widehat{N}_{B}\left(y+t w^{\prime}\right) \\
& \subseteq \limsup _{\substack{t \downarrow 0 \\
d^{\prime} \rightarrow d}} \widehat{N}_{A}\left(x+t d^{\prime}\right) \times \limsup _{\substack{t \downarrow 0 \\
w^{\prime} \rightarrow w}} \widehat{N}_{B}\left(y+t w^{\prime}\right) \\
& =N_{A}(x ; d) \times N_{B}(y ; w) .
\end{aligned}
$$

Conversely, take $(p, q) \in N_{A}(x ; d) \times N_{B}(y ; w)$. Without loss of generality, assume that $A$ is regular at $x$ in direction $d$. Since $q \in N_{B}(y ; w)$, there exists $t_{n} \downarrow 0$ and $w_{n} \rightarrow w$ and $q_{n} \rightarrow q$ such that $q_{n} \in \widehat{N}_{B}\left(y+t_{n} w_{n}\right)$. Since $p \in N_{A}(x ; d)$ and $A$ is regular at $x$ in direction $d$, for the above $t_{n}$ there exist $d_{n} \rightarrow d$ and $p_{n} \rightarrow p$ such that $p_{n} \in \widehat{N}_{A}\left(x+t_{n} d_{n}\right)$. So

$$
\left(p_{n}, q_{n}\right) \in \widehat{N}_{A}\left(x+t_{n} d_{n}\right) \times \widehat{N}_{B}\left(y+t_{n} w_{n}\right)=\widehat{N}_{A \times B}\left((x, y)+t_{n}\left(d_{n}, w_{n}\right)\right) .
$$

By definition, this means that $(p, q) \in N_{A \times B}((x, y) ;(d, w))$.

Now suppose that $A$ and $B$ are regular in direction $d, w$ respectively. Then

$$
\begin{aligned}
N_{A \times B}((x, y) ;(d, w)) & \subseteq N_{A}(x ; d) \times N_{B}(y ; w) \\
& =N_{A}^{i}(x ; d) \times N_{B}^{i}(y ; w) \\
& =N_{A \times B}^{i}((x, y) ;(d, w)) .
\end{aligned}
$$

Therefore $A \times B$ is regular in direction $(d, w)$.

Proposition 3.3 Let $\left(z_{1}, \ldots, z_{I}\right) \in A_{1} \times \cdots \times A_{I}$ and $\left(d_{1}, \ldots, d_{I}\right)$ be given. Then

$$
\begin{aligned}
& T_{A_{1} \times \cdots \times A_{I}}\left(z_{1}, \ldots, z_{I}\right) \subseteq T_{A_{1}}\left(z_{1}\right) \times \cdots \times T_{A_{I}}\left(z_{I}\right), \\
& N_{A_{1} \times \cdots \times A_{I}}\left(\left(z_{1}, \ldots, z_{I}\right) ;\left(d_{1}, \ldots, d_{I}\right)\right) \subseteq N_{A_{1}}\left(z_{1} ; d_{1}\right) \times \cdots \times N_{A_{I}}\left(z_{I} ; d_{I}\right),
\end{aligned}
$$

and equality holds if all except at most one of $A_{i}$ for $i=1, \ldots, I$ are directionally regular at $z_{i}$. 
Proof. By Corollary 3.1, the directional regularity implies the geometric derivability. Then the tangent set formula follows from applying [16, Proposition 1]. The directional normal cone formula follows from Proposition 3.2 .

In the rest of this section we will study some calculus rule of the directional normal cone, and in the mean time examine the directional regularity.

Proposition 3.4 If $C \subseteq \mathbb{R}^{n}$ is a closed cone, then

$$
N_{C}^{i}(0 ; d)=N_{C}(0 ; d)=N_{C}(d), \forall d \in \mathbb{R}^{n} .
$$

Proof. Since $C$ is a cone, we have $T_{C}(0)=C$. If $d \notin C$, then

$$
N_{C}^{i}(0 ; d)=N_{C}(0 ; d)=N_{C}(d)=\emptyset \text {. }
$$

If $d \in C$, then

$$
\left.N_{C}(0 ; d)=\limsup _{\substack{t \downarrow 0 \\ d^{\prime} \rightarrow d}} \widehat{N}_{C}\left(t d^{\prime}\right)=\limsup _{\substack{d^{\prime} \rightarrow d \\ N_{C}}} \widehat{N}^{\prime}\right)=N_{C}(d) .
$$

Now we show that $N_{C}^{i}(0 ; d)=N_{C}(0 ; d)$. It suffices to show $N_{C}(0 ; d) \subseteq N_{C}^{i}(0 ; d)$. Take $v \in N_{C}(0 ; d)$. Then there exists $\eta_{n} \downarrow 0$ and $d_{n} \rightarrow d$ and $v_{n} \rightarrow v$ with $v_{n} \in \widehat{N}_{C}\left(\eta_{n} d_{n}\right)$. For any $t_{n} \downarrow 0$, take $d_{n}$ and $v_{n}$ above, then $d_{n} \rightarrow d$ and $v_{n} \rightarrow v$ with $v_{n} \in \widehat{N}_{C}\left(\eta_{n} d_{n}\right)=\widehat{N}_{C}\left(d_{n}\right)=$ $\widehat{N}_{C}\left(t_{n} d_{n}\right)$. Hence $v \in N_{C}^{i}(0 ; d)$.

We next show that any convex set is regular along any direction.

Proposition 3.5 Any closed convex set $A$ is directionally regular.

Proof. Since $N_{A}^{i}(z ; d) \subseteq N_{A}(z ; d)$ for any $z$ and $d$, it suffices to prove $N_{A}(z ; d) \subseteq N_{A}^{i}(z ; d)$ for any $d \in T_{A}(z)$. Take $w \in N_{A}(z ; d)$ with $d \in T_{A}(z)$. Then there exists $\eta_{k} \downarrow 0, d_{k} \rightarrow d$ and $w_{k} \rightarrow w$ with $w_{k} \in \widehat{N}_{A}\left(z+\eta_{k} d_{k}\right)$. Since $A$ is convex, it follows that

$$
\left\langle w_{k}, z^{\prime}-z-\eta_{k} d_{k}\right\rangle \leq 0, \quad \forall z^{\prime} \in A .
$$

In particular, taking $z^{\prime}=z$ in the above, we have

$$
\left\langle w_{k}, d_{k}\right\rangle \geq 0 \text {. }
$$

Let $t_{n} \downarrow 0$. Then since $\eta_{k} \downarrow 0$, for each fixed $n$, there exists $k(n)$ satisfying $k(n) \geq n$ and $\eta_{k(n)}<t_{n}$. Hence $k(n) \rightarrow \infty$ as $n \rightarrow \infty$. For simplicity, denote by $d_{n}:=d_{k(n)}$ and $w_{n}:=w_{k(n)}$. Since $\left\{d_{n}\right\}$ and $\left\{w_{n}\right\}$ are subsequences of $\left\{d_{k}\right\}$ and $\left\{w_{k}\right\}$ respectively, we have $d_{n} \rightarrow d$ and $w_{n} \rightarrow w$. Hence for all $z^{\prime} \in A$ we have

$$
\begin{aligned}
\left\langle w_{n}, z^{\prime}-z-t_{n} d_{n}\right\rangle & =\left\langle w_{k(n)}, z^{\prime}-z-t_{n} d_{k(n)}\right\rangle \\
& =\left\langle w_{k(n)}, z^{\prime}-z-\eta_{k(n)} d_{k(n)}\right\rangle+\left\langle w_{k(n)}, \eta_{k(n)} d_{k(n)}-t_{n} d_{k(n)}\right\rangle \\
& \leq\left\langle w_{k(n)}, \eta_{k(n)} d_{k(n)}-t_{n} d_{k(n)}\right\rangle \\
& \leq 0
\end{aligned}
$$

where the first inequality comes from (9) and the second inequality follows from (10). So $w_{n} \in N_{A}\left(z+t_{n} d_{n}\right)=\widehat{N}_{A}\left(z+t_{n} d_{n}\right)$. By the definition of the inner limiting normal cone, we have $w \in N_{A}^{i}(z ; d)$. This completes the proof.

Based on ([6), Propositions 3.3 and [3.5, we can obtain the following results. 
Corollary 3.2 Let $A_{i}$ be given for $i=1, \ldots, I$.

(i) If $A_{i}$ is regular at $z_{i} \in A_{i}$ in direction $d_{i}$ for $i=1, \ldots, I$, then $A_{1} \times \cdots \times A_{I}$ is regular at $\left(z_{1}, \ldots, z_{I}\right)$ in direction $\left(d_{1}, \ldots, d_{I}\right)$. Moreover, (8) holds as an equation.

(ii) If $A_{i}$ is directionally regular at $z_{i} \in A_{i}$ for $i=1, \ldots, I$, then $A_{1} \times \cdots \times A_{I}$ is directionally regular at $\left(z_{1}, \ldots, z_{I}\right)$. Moreover (7) and (8) holds as an equation for all $d_{i}$.

(iii) If $A_{i}$ is closed and convex for $i=1, \ldots, I$, then $A_{1} \times \cdots \times A_{I}$ is directionally regular. Moreover (7) and (8) holds as an equation for all $z_{i} \in A_{i}$ and $d_{i}$.

Corollary [3.2 (iii) extends the result given in [15, Lemma 1], where each $A_{i}$ is assumed to be a polyhedral convex set. In Section 6, we will show that the second-order cone complementarity set, although it is a nonconvex set, is directionally regular.

\section{Sufficient conditions for the error bound property via di- rectional normal cones}

Consider a general system in the form: $P(z) \in D$, where $P: \mathbb{R}^{l} \rightarrow \mathbb{R}^{s}$ and $D \subseteq \mathbb{R}^{s}$ is closed. We say that the system $P(z) \in D$ has a local error bound at $z$ such that $P(z) \in D$, or the set-valued mapping $M(z):=P(z)-D$ is metrically subregular at $(z, 0) \in \operatorname{gph} M$, if there exist a neighborhood $V$ of $z$ and a positive number $\kappa>0$ such that

$$
d_{M^{-1}(0)}\left(z^{\prime}\right) \leq \kappa d_{D}\left(P\left(z^{\prime}\right)\right), \quad \forall z^{\prime} \in V .
$$

It is easy to see that $M$ is metrically subregular at $(z, 0)$ if and only if its inverse set-valued mapping $M^{-1}$ is calm at $(0, z) \in \operatorname{gph} M^{-1}$, i.e., there exist a neighborhood $W$ of 0 , a neighborhood $V$ of $z$ and a positive number $\kappa>0$ such that

$$
M^{-1}(w) \cap V \subseteq M^{-1}(0)+\kappa\|w\| \bar{B}, \quad \forall w \in W .
$$

The metric subregularity is obviously weaker than the metric regularity (or the pseudo Lipschitz continuity) which ensures the existence of a neighborhood $W$ of 0 , a neighborhood $V$ of $z$ and a positive number $\kappa>0$ such that

$$
M^{-1}(w) \cap V \subseteq M^{-1}\left(w^{\prime}\right)+\kappa\left\|w-w^{\prime}\right\| \bar{B}, \quad \forall w, w^{\prime} \in W .
$$

While the term for the calmness of a set-valued map was first coined in [30], it was introduced as the pseudo-upper Lipschitz continuity in [35], taking into the account that it is weaker than both the pseudo-Lipschitz continuity of Aubin [2] and the upper Lipschitz continuity of Robinson [27, 28]. More information and discussion on metric regularity and the related concept can be found in [21].

Recall that the following well-known criteria for metric regularity of the set-valued mapping $M$ or the Aubin property of its inverse mapping $M^{-1}(w)=\left\{z \in \mathbb{R}^{l} \mid P(z)-w \in D\right\}$.

Theorem 4.1 (see e.g. [30]) Consider the system $P(z) \in D$, where $P$ is smooth and $D$ is closed. Then the set-valued map $M(z):=P(z)-D$ is metrically regular at $(z, 0)$ if and only if the no nonzero abnormal multiplier constraint qualification (NNAMCQ) holds at $z$, i.e.,

$$
\nabla P(z)^{T} \lambda=0, \lambda \in N_{D}(P(z)) \quad \Longrightarrow \quad \lambda=0 .
$$


While following [34, the condition (11) is called NNAMCQ, there are other terminologies in the literature; e.g., generalized MFCQ (GMFCQ) in [11] and Mordukhovich criterion in [15]. This condition is a necessary and sufficient condition for metric regularity and hence may be too strong for metric subregularity.

By using the directional normal cone instead of the limiting normal cone, the following sufficient conditions for metric subregularity have been introduced.

Theorem 4.2 ([15, Corollary 1]) Let $P(z) \in D$ with $P$ smooth. The set-valued mapping $M(z):=P(z)-D$ is metrically subregular at $(z, 0)$ if the first-order sufficient condition for metric subregularity (FOSCMS) holds: for every $0 \neq w$ such that $\nabla P(z) w \in T_{D}(P(z))$ one has

$$
\nabla P(z)^{T} \lambda=0, \lambda \in N_{D}(P(z) ; \nabla P(z) w) \quad \Longrightarrow \quad \lambda=0 .
$$

Let us discuss the relation between FOSCMS and NNAMCQ. FOSCMS can be rewritten equivalently as

$$
\nabla P(z)^{T} \lambda=0, \quad \lambda \in \bigcup_{w \in \Gamma} N_{D}(P(z) ; \nabla P(z) w) \Longrightarrow \lambda=0,
$$

where $\Gamma:=\left\{w \neq 0 \mid \nabla P(z) w \in T_{D}(P(z))\right\}$. Noth that FOSCMS holds automatically if $\Gamma=\emptyset$, i.e.,

$$
\nabla P(z) w \in T_{D}(P(z)) \Longrightarrow w=0 .
$$

According to the graphical derivative criterion for strong metric subregularity [8], condition (13) is equivalent to saying that the set-valued map $M(z)=P(z)-D$ is strongly metrically subregular (or equivalently its inverse is isolatedly calm) at $(z, 0)$.

Theorem 4.3 Let $M(z):=P(z)-D$ and $(z, 0) \in \operatorname{gph} M . \quad F O S C M S$ at $z$ is equivalent to $N N A M C Q$ at $z$ under one of the following assumptions:

(i) $\nabla P(z)$ does not have full column rank;

(ii) $D$ is a closed and convex set and there exists $w \neq 0$ such that $\nabla P(z) w \in T_{D}(P(z))$.

Proof. (i). If $\nabla P(z)$ does not have full column rank, then there exists $\bar{w} \neq 0$ such that $\nabla P(z) \bar{w}=0$. So $\Gamma \neq \emptyset$. Since

$$
N_{D}(P(z) ; \nabla P(z) \bar{w})=N_{D}(P(z) ; 0)=N_{D}(P(z)),
$$

we have

$$
\bigcup_{w \in \Gamma} N_{D}(P(z) ; \nabla P(z) w)=N_{D}(P(z))
$$

It follows that FOSCMS and NNAMCQ are equivalent by comparing the conditions (11) and (12).

(ii). Suppose that $D$ is a closed and convex set and there exists $w \neq 0$ such that $\nabla P(z) w \in T_{D}(P(z))$. Then FOSCMS means (12) holds. Since the directional normal cone is in general a subset of the limiting normal cone, it is clear that NNAMCQ implies FOSCMS. Conversely assume that FOSCMS holds. Take $\lambda$ satisfying $\nabla P(z)^{T} \lambda=0$ and $\lambda \in N_{D}(P(z))$. Note that $\langle\lambda, \nabla P(z) w\rangle=\left\langle\nabla P(z)^{T} \lambda, w\right\rangle=0$. Hence $\lambda \in N_{D}(P(z)) \cap(\nabla P(z) w)^{\perp}$, which means that $\lambda \in N_{D}(P(z) ; \nabla P(z) w)$ by [14, Lemma 2.1]. The FOSCMS at $z$ then ensures $\lambda=0$. Hence NNAMCQ holds at $z$. 
Remark 4.1 The assumptions (i) or (ii) given in Theorem 4.3 cannot be omitted. For example, when $\nabla P(z)$ has full column rank and $D$ is nonconvex with $\Gamma \neq \emptyset$, FOSCMS may be strictly weaker than NNAMCQ; see Example 4.1 below.

Example 4.1 Consider the optimization problem:

$$
\begin{array}{cl}
\min & z_{1}+z_{2} \\
\text { s.t. } & \left(z_{1}, z_{2}\right) \in \mathcal{K} \\
& \left(z_{1}, z_{2}\right) \in \Omega,
\end{array}
$$

where $\mathcal{K}:=\left\{\left(z_{1}, z_{2}\right) \in \mathbb{R}^{2}\left|z_{1} \geq\right| z_{2} \mid\right\}$ and $\Omega:=\left\{\left(z_{1}, z_{2}\right) \in \mathbb{R}^{2} \mid z_{1} \geq 0, z_{2} \geq 0, z_{1} z_{2}=0\right\}$. Denote by $P(z)=(z, z)$ and $D=\mathcal{K} \times \Omega$. The optimal solution is $z^{*}=(0,0)$. It is clear that

$$
\left\{w \neq 0 \mid \nabla P\left(z^{*}\right) w \in T_{D}\left(P\left(z^{*}\right)\right)\right\}=\{w \neq 0 \mid(w, w) \in D\}=\left\{\left(w_{1}, w_{2}\right) \mid w_{1}>0, w_{2}=0\right\},
$$

$\nabla P\left(z^{*}\right)$ has a full column rank and $D$ is nonconvex. By virtue of Proposition 3.4, since D is a cone we have $N_{D}\left((0,0) ; \nabla P\left(z^{*}\right) w\right)=N_{D}\left(\nabla P\left(z^{*}\right) w\right)$, and hence the condition

$$
\nabla P\left(z^{*}\right)^{T} \lambda=0, \quad \lambda \in N_{D}\left(P\left(z^{*}\right) ; \nabla P\left(z^{*}\right) w\right)=N_{D}\left(\nabla P\left(z^{*}\right) w\right)=N_{\mathcal{K}}\left(w_{1}, w_{2}\right) \times N_{\Omega}\left(w_{1}, w_{2}\right)
$$

with $w_{1}>0, w_{2}=0$ takes the form

$$
\lambda^{\mathcal{K}}+\lambda^{\Omega}=0, \quad\left(\lambda^{\mathcal{K}}, \lambda^{\Omega}\right) \in\{(0,0)\} \times(\{0\} \times \mathbb{R}),
$$

which implies that $\left(\lambda^{\mathcal{K}}, \lambda^{\Omega}\right)=(0,0)$. Hence FOSCMS holds at $z^{*}$.

On the other hand,

$$
\nabla P\left(z^{*}\right)^{T} \lambda=0, \quad \lambda=\left(\lambda^{\mathcal{K}}, \lambda^{\Omega}\right) \in N_{D}\left(P\left(z^{*}\right)\right)
$$

takes the form

$$
\lambda^{\mathcal{K}}+\lambda^{\Omega}=0, \quad\left(\lambda^{\mathcal{K}}, \lambda^{\Omega}\right) \in-\mathcal{K} \times N_{\Omega}(0,0) .
$$

Take $\lambda^{\mathcal{K}}=(-1,0)$ and $\lambda^{G}=(1,0)$. Then $\lambda^{\mathcal{K}} \in-\mathcal{K}$ and $\lambda^{\Omega} \in N_{\Omega}(0,0)$. Hence NNAMCQ does not hold at $z^{*}$.

It is interesting to note that each of the set-valued mappings for the two split systems $M_{1}(z):=\left(z_{1}, z_{2}\right)-\mathcal{K}$ and $M_{2}(z):=\left(z_{1}, z_{2}\right)-\Omega$ are both metrically regular at $\left(z^{*}, 0\right)$, but the one for the whole system $M(z)=(z, z)-\mathcal{K} \times \Omega$ is only metrically subregular (not metrically regular) at $\left(z^{*}, 0\right)$.

In many situations, the constraint system $P(z) \in D$ can be split into subsystems $P_{1}(z) \in$ $D_{1}, P_{2}(z) \in D_{2}$ such that one subsystem can be checked to have error bound property easily. In Klatte and Kummer [21, Theorem 2.5], it is shown that if both $M_{1}^{-1}$ and $M_{2}^{-1}$ are calm at $\left(0, z^{*}\right)$ and $M_{2}$ is pseudo-Lipschitz continuous at $\left(z^{*}, 0\right)$, then checking the calmness of the intersection $M^{-1}(\alpha, \beta):=M_{1}^{-1}(\alpha) \cap M_{2}^{-1}(\beta)$ at $\left(0,0, z^{*}\right)$ can be reduced to checking the calmness of $H(\beta):=M_{1}^{-1}(0) \cap M_{2}^{-1}(\beta)$ at $\left(0, z^{*}\right)$. In Example 4.1, both $M_{1}^{-1}$ and $M_{2}^{-1}$ are calm at $\left(0, z^{*}\right)$ and $M_{2}$ can be checked to be pseudo-Lipschitz continuous at $\left(z^{*}, 0\right)$ by using Mordukhovich criterion, and $H(\beta)=\{z \mid z \in \mathcal{K}, z-\beta \in \Omega\}$ is calm at $\left(0, z^{*}\right)$ as a polyhedral multifunction. This ensures the calmness of $M^{-1}$, or equivalently, the metric subregularity of the whole system $M(z)=P(z)-D$.

In [16. Theorem 2], the first order sufficient condition for metric subregularity for a split system with product of two sets is given. When one of the subsystem is known to be metrically subregular, the condition given in [16, Theorem 2] is completely verifiable using the initial data of the problem. We now extend this result to the product of finitely many sets. 
Theorem 4.4 Let $P\left(z^{*}\right) \in D$ and assume that $P$ and $D$ can be written in the form

$$
P(z)=\left(P_{1}(z), P_{2}(z), \ldots, P_{I}(z)\right), \quad D=D_{1} \times D_{2} \times \cdots \times D_{I},
$$

where $P_{i}: \mathbb{R}^{n} \rightarrow \mathbb{R}^{s_{i}}$ are smooth and $D_{i} \subseteq \mathbb{R}^{s_{i}}, i=1,2, \ldots, I$, are closed such that the set-valued map $M_{1}(z):=P_{1}(z)-D_{1}$ is metrically subregular at $\left(z^{*}, 0\right)$. Further assume that for every $0 \neq w$ such that $\nabla P_{i}\left(z^{*}\right) w \in T_{D_{i}}\left(P_{i}\left(z^{*}\right)\right), i=1,2, \ldots, I$, one has

$$
\left.\begin{array}{l}
\nabla P_{1}\left(z^{*}\right)^{T} \lambda^{1}+\sum_{i=2}^{I} \nabla P_{i}\left(z^{*}\right)^{T} \lambda^{i}=0, \\
\lambda^{i} \in N_{D_{i}}\left(P_{i}\left(z^{*}\right) ; \nabla P_{i}\left(z^{*}\right) w\right) \forall i=1,2, \ldots, I
\end{array}\right\} \Longrightarrow \lambda^{i}=0 \quad \forall i=2, \ldots, I .
$$

Then the set-valued mapping $M(z):=P(z)-D$ is metrically subregular at $\left(z^{*}, 0\right)$. Moreover, if all $D_{i}$ except at most $D_{1}$ are directionally regular at $P_{i}\left(z^{*}\right)$, then (14) is equivalent to that for every $w \neq 0$ such that $\nabla P\left(z^{*}\right) w \in T_{D}\left(P\left(z^{*}\right)\right)$, one has

$$
\begin{aligned}
& \left\{\begin{array}{l}
\nabla P_{1}\left(z^{*}\right)^{T} \lambda^{1}+\sum_{i=2}^{I} \nabla P_{i}\left(z^{*}\right)^{T} \lambda^{i}=0, \\
\lambda^{1} \in N_{D_{1}}\left(P_{1}\left(z^{*}\right) ; \nabla P_{1}\left(z^{*}\right) w\right), \\
\left(\lambda^{2}, \ldots, \lambda^{I}\right) \in N_{D_{2} \times \cdots \times D_{I}}\left(\left(P_{2}\left(z^{*}\right), \ldots, P_{I}\left(z^{*}\right)\right) ; \nabla P_{2}\left(z^{*}\right) w, \ldots, \nabla P_{I}\left(z^{*}\right) w\right)
\end{array}\right. \\
& \Longrightarrow \lambda^{i}=0 \forall i=2, \ldots, I .
\end{aligned}
$$

Proof. Let $w \neq 0$ satisfying $\nabla P\left(z^{*}\right) w \in T_{D}\left(P\left(z^{*}\right)\right)$ such that (15) holds. Then by Proposition 3.3. we have $\nabla P_{i}\left(z^{*}\right) w \in T_{D_{i}}\left(P_{i}\left(z^{*}\right)\right)$, and $\lambda^{i} \in N_{D_{i}}\left(P_{i}\left(z^{*}\right) ; \nabla P_{i}\left(z^{*}\right) w\right)$ for $i=1,2, \ldots, I$. Since (14) holds at $z^{*}$, it follows that $\lambda^{i}=0$ for $i=2, \ldots, I$. Applying [16. Theorem 2], we have that the set-valued mapping $M(z):=P(z)-D$ is metrically subregular at $\left(z^{*}, 0\right)$. Moreover suppose that all $D_{i}$ except at most $D_{1}$ are directionally regular at $P_{i}\left(z^{*}\right)$. Then by Proposition 3.3 ,

$$
\begin{aligned}
& T_{D}\left(P\left(z^{*}\right)\right)=T_{D_{1}}\left(P_{1}\left(z^{*}\right)\right) \times \cdots \times T_{D_{I}}\left(P_{I}\left(z^{*}\right)\right), \\
& N_{D_{2} \times \cdots \times D_{I}}\left(\left(P_{2}\left(z^{*}\right), \ldots, P_{I}\left(z^{*}\right)\right) ; \nabla P_{2}\left(z^{*}\right) w, \ldots, \nabla P_{I}\left(z^{*}\right) w\right) \\
& =N_{D_{2}}\left(P_{2}\left(z^{*}\right) ; \nabla P_{2}\left(z^{*}\right) w\right) \times \cdots \times N_{D_{I}}\left(P_{I}\left(z^{*}\right) ; \nabla P_{I}\left(z^{*}\right) w\right),
\end{aligned}
$$

and hence the two conditions are equivalent.

\section{Expressions for tangent cones}

In order to use the sufficient conditions for metric subregularity in terms of directional normal cones, one needs to derive the formula for the tangent cone involved. In this section we derive the exact expressions for the tangent cone of the second-order cone complementarity set. Moreover we show that it is geometrically derivable.

The following formula for the tangent cone of the second-order cone is well-known.

Proposition 5.1 [3, Lemma 25] Let $\mathcal{K}$ be the m-dimensional second-order cone.

$$
T_{\mathcal{K}}(x)=\left\{\begin{array}{ll}
\mathbb{R}^{m} & \text { if } x \in \operatorname{int} \mathcal{K} \\
\mathcal{K} & \text { if } x=0 \\
d \in \mathbb{R}^{m}:-d_{1}+\bar{x}_{2}^{T} d_{2} \leq 0 & \text { if } x \in \operatorname{bd} \mathcal{K} \backslash\{0\}
\end{array}\right\} .
$$


Let $\mathcal{K}$ be the $m$-dimensional second-order cone and

$$
\Omega=\{(x, y) \mid \mathcal{K} \ni x \perp y \in \mathcal{K}\}
$$

be the corresponding second-order cone complementarity set. In what follows we show that the set $\Omega$ is geometrically derivable and give a characterization in terms of the metric projection operator. The characterization of the tangent cone was also given in 20 , Proposition $3.1]$.

Proposition 5.2 The set $\Omega$ is geometrically derivable, and for any $(x, y) \in \Omega$,

$$
T_{\Omega}(x, y)=T_{\Omega}^{i}(x, y)=\left\{(d, w) \mid \Pi_{\mathcal{K}}^{\prime}(x-y ; d-w)=d\right\} .
$$

Proof. Since $T_{\Omega}^{i}(x, y) \subseteq T_{\Omega}(x, y)$, it suffices to prove

$$
T_{\Omega}(x, y) \subseteq \Upsilon(x, y) \subseteq T_{\Omega}^{i}(x, y),
$$

where $\Upsilon(x, y):=\left\{(d, w) \mid \Pi_{\mathcal{K}}^{\prime}(x-y ; d-w)=d\right\}$. Take $(d, w) \in T_{\Omega}(x, y)$. Then by definition, there exist $t_{n} \downarrow 0$ and $\left(d_{n}, w_{n}\right) \rightarrow(d, w)$ such that $(x, y)+t_{n}\left(d_{n}, w_{n}\right) \in \Omega$. By (44), we have

$$
\Pi_{\mathcal{K}}\left(x+t_{n} d_{n}-y-t_{n} w_{n}\right)=x+t_{n} d_{n}=\Pi_{\mathcal{K}}(x-y)+t_{n} d_{n}
$$

Hence

$$
\Pi_{\mathcal{K}}^{\prime}(x-y ; d-w)=\lim _{t_{n} \downarrow 0} \frac{\Pi_{\mathcal{K}}\left(x+t_{n} d_{n}-y-t_{n} w_{n}\right)-\Pi_{\mathcal{K}}(x-y)}{t_{n}}=d .
$$

Therefore $T_{\Omega}(x, y) \subseteq \Upsilon(x, y)$. Now take $(d, w) \in \Upsilon(x, y)$. Then for any given $t_{n} \downarrow 0$

$$
\Pi_{\mathcal{K}}\left(x-y+t_{n}(d-w)\right)-\Pi_{\mathcal{K}}(x-y)=t_{n} \Pi_{\mathcal{K}}^{\prime}(x-y ; d-w)+o\left(t_{n}\right)=t_{n} d+o\left(t_{n}\right),
$$

i.e.,

$$
\Pi_{\mathcal{K}}\left(x-y+t_{n}(d-w)\right)=\Pi_{\mathcal{K}}(x-y)+t_{n} d+o\left(t_{n}\right) .
$$

Hence

$$
\begin{aligned}
& \left(\Pi_{\mathcal{K}}(x-y)+t_{n} d+o\left(t_{n}\right), \Pi_{\mathcal{K}}(x-y)+t_{n} d+o\left(t_{n}\right)-\left(x-y+t_{n}(d-w)\right)\right) \in \Omega \\
\Longleftrightarrow & \left(x+t_{n} d+o\left(t_{n}\right), y+t_{n} w+o\left(t_{n}\right)\right) \in \Omega .
\end{aligned}
$$

This means $(d, w) \in \liminf _{t_{n} \downarrow 0} \frac{\Omega-(x, y)}{t_{n}}$. Hence $\Upsilon(x, y) \subseteq T_{\Omega}^{i}(x, y)$.

With the above result, an explicit expression of tangent cone $T_{\Omega}$ is also given in 20, Theorem 3.1]. However, in that explicit formula, for the case where $x-y \notin \mathcal{K} \cup \mathcal{K}^{\circ}$, the directional derivative of the projection operator is involved. In the hope that only the initial data on $x, y$ is used, we next provide another explicit expression for $T_{\Omega}(x, y)$ without involving $\Pi_{\mathcal{K}}^{\prime}(x-y)$. 
Theorem 5.1 Let $\Omega$ be defined as in (16). Then for any $(x, y) \in \Omega$,

$$
\begin{aligned}
& T_{\Omega}^{i}(x, y)=T_{\Omega}(x, y) \\
& =\left\{\begin{array}{l|ll}
d=0, w \in \mathbb{R}^{m}, & \text { if } x=0, y \in \operatorname{int} \mathcal{K} ; \\
d \in \mathbb{R}^{m}, w=0, & \text { if } x \in \operatorname{int} \mathcal{K}, y=0 ; \\
x_{1} \hat{w}-y_{1} d \in \mathbb{R} x, d \perp y, w \perp x, & \text { if } x, y \in \mathrm{bd} \mathcal{K} \backslash\{0\} ; \\
d=0, w \in T_{\mathcal{K}}(y) \text { or } d \in \mathbb{R}_{+} \hat{y}, w \perp \hat{y}, & \text { if } x=0, y \in \mathrm{bd} \mathcal{K} \backslash\{0\} ; \\
d \in T_{\mathcal{K}}(x), w=0 \text { or } d \perp \hat{x}, w \in \mathbb{R}_{+} \hat{x}, & \text { if } x \in \mathrm{bd} \mathcal{K} \backslash\{0\}, y=0 ; \\
d \in \mathcal{K}, w \in \mathcal{K}, d \perp w, & \text { if } x=0, y=0 .
\end{array}\right\} .
\end{aligned}
$$

Proof. Note that $\Pi_{\mathcal{K}}(x-y)$ is continuously differentiable at $x-y$, provided that $x=0$ and $y \in \operatorname{int} \mathcal{K}$, or $x \in \operatorname{int} \mathcal{K}$ and $y=0$, or $x, y \in \operatorname{bd} \mathcal{K} \backslash\{0\}$ with $x^{T} y=0$. Hence $D^{*} \Pi_{\mathcal{K}}(x-y)=$ $\nabla \Pi_{\mathcal{K}}(x-y)$ in the above cases, which in turn implies

$$
\Pi_{\mathcal{K}}^{\prime}(x-y ; d-w)=d \Longleftrightarrow D^{*} \Pi_{\mathcal{K}}(x-y)(d-w)=d \Longleftrightarrow(w,-d) \in N_{\Omega}(x, y),
$$

where the second equivalence is due to Proposition 2.4. By the expression of the limiting normal cone in Proposition 2.6, we have the following conclusions.

Case 1: If $x=0$ and $y \in \operatorname{int} \mathcal{K}$, then $w \in \mathbb{R}^{m}$ and $d=0$.

Case 2: If $x \in \operatorname{int} \mathcal{K}$ and $y=0$, then $d \in \mathbb{R}^{m}$ and $w=0$.

Case 3: If $x, y \in \mathrm{bd} \mathcal{K} \backslash\{0\}$ with $x^{T} y=0$, then

$$
x_{1} \hat{w}-y_{1} d \in \mathbb{R} x, \quad d \perp y, w \perp x .
$$

Case 4: If $x=0$ and $y \in \mathrm{bd} \mathcal{K} \backslash\{0\}$, since by Proposition [5.2, $(d, w) \in T_{\Omega}(x, y)$ is equivalent to saying that $\Pi_{\mathcal{K}}^{\prime}(x-y ; d-w)=d$. According to the formula of directional derivative for $\Pi_{\mathcal{K}}$ in Proposition 2.2(iii), we have $(d, w) \in T_{\Omega}(x, y)$ if and only if

$$
\frac{1}{2}\left(d_{1}-w_{1}-\bar{y}_{2}^{T} d_{2}+\bar{y}_{2}^{T} w_{2}\right)_{+}\left[\begin{array}{c}
1 \\
-\bar{y}_{2}
\end{array}\right]=\left[\begin{array}{l}
d_{1} \\
d_{2}
\end{array}\right]
$$

We now claim that the set of solutions to equation (17) is

$$
\left\{(d, w) \mid d=0, w \in T_{\mathcal{K}}(y) \text { or } d \in \mathbb{R}_{+} \hat{y}, w \perp \hat{y}\right\} .
$$

By definition of the tangent cone, $(d, w) \in T_{\Omega}(x, y)$ if and only if there exists $\left(d_{n}, w_{n}\right) \rightarrow$ $(d, w)$ and $t_{n} \rightarrow 0$ with $t_{n} \geq 0$ satisfying $\left(t_{n} d_{n}, y+t_{n} w_{n}\right)=(x, y)+t_{n}\left(d_{n}, w_{n}\right) \in \Omega$, i.e.,

$$
t_{n} d_{n} \in \mathcal{K}, \quad y+t_{n} w_{n} \in \mathcal{K}, \quad t_{n} d_{n} \perp y+t_{n} w_{n},
$$

which implies

$$
d \in \mathcal{K}, \quad w \in T_{\mathcal{K}}(y), \quad d \perp y .
$$

Note that $d \in \mathcal{K}$ and $\langle y, d\rangle=0$ implies that either $d=0$ or $d \in \mathbb{R}_{++} \hat{y}$. If $d=0$, then (17) takes the form as

$$
\frac{1}{2}\left(-w_{1}+\bar{y}_{2}^{T} w_{2}\right)_{+}\left[\begin{array}{c}
1 \\
-\bar{y}_{2}
\end{array}\right]=\left[\begin{array}{l}
0 \\
0
\end{array}\right],
$$


which implies $-w_{1}+\bar{y}_{2}^{T} w_{2} \leq 0$, i.e., $w \in T_{\mathcal{K}}(y)$ by the formula of tangent cone of $\mathcal{K}$ in Proposition [5.1] If $d \in \mathbb{R}_{++} \hat{y}$, then $d=\tau \hat{y}=\tau\left(y_{1},-y_{2}\right)$ for some $\tau>0$. Hence

$$
\bar{y}_{2}^{T} d_{2}=\bar{y}_{2}^{T}\left(-\tau y_{2}\right)=-\tau\left\|y_{2}\right\|=-\tau y_{1}=-d_{1} .
$$

It then follows from (17) that

$$
d_{1}=\frac{1}{2}\left(d_{1}-w_{1}-\bar{y}_{2}^{T} d_{2}+\bar{y}_{2}^{T} w_{2}\right)_{+}=\frac{1}{2}\left(2 d_{1}-w_{1}+\bar{y}_{2}^{T} w_{2}\right)_{+},
$$

which implies $-w_{1}+\bar{y}_{2}^{T} w_{2}=0$, i.e., $w \perp \hat{y}$. Thus, $(d, w)$ satisfies (18).

Conversely, if $d=0$ and $w \in T_{\mathcal{K}}(y)$, then $-w_{1}+\bar{y}_{2}^{T} w_{2} \leq 0$ by Proposition [5.1, and hence

$$
\left(d_{1}-w_{1}-\bar{y}_{2}^{T} d_{2}+\bar{y}_{2}^{T} w_{2}\right)_{+}=\left(-w_{1}+\bar{y}_{2}^{T} w_{2}\right)_{+}=0,
$$

which implies that (17) holds, i.e., $(d, w) \in T_{\Omega}(x, y)$. For the other case, if $d \in \mathbb{R}_{++} \hat{y}$ and $w \perp \hat{y}$, then $d=\tau \hat{y}$ for some $\tau>0$ and $w_{1}=\bar{y}_{2}^{T} w_{2}$. Therefore

$$
\begin{aligned}
\frac{1}{2}\left(d_{1}-w_{1}-\bar{y}_{2}^{T} d_{2}+\bar{y}_{2}^{T} w_{2}\right)_{+}\left[\begin{array}{c}
1 \\
-\bar{y}_{2}
\end{array}\right] & =\frac{1}{2}\left(d_{1}-\bar{y}_{2}^{T} d_{2}\right)_{+}\left[\begin{array}{c}
1 \\
-\bar{y}_{2}
\end{array}\right] \\
& =d_{1}\left[\begin{array}{c}
1 \\
-\bar{y}_{2}
\end{array}\right] \\
& =\left[\begin{array}{c}
d_{1} \\
d_{2}
\end{array}\right],
\end{aligned}
$$

where the second equation is due to $d_{1}-\bar{y}_{2}^{T} d_{2}=d_{1}+\tau \bar{y}_{2}^{T} y_{2}=d_{1}+\tau\left\|y_{2}\right\|=d_{1}+\tau y_{1}=2 d_{1}$ and the third equation comes from $-d_{1} \bar{y}_{2}=-\tau y_{1} \bar{y}_{2}=-\tau y_{2}=d_{2}$. This means that (17) holds, i.e., $(d, w) \in T_{\Omega}(x, y)$. In summary, we have shown that

$$
T_{\Omega}(x, y)=\left\{(d, w) \mid d=0, w \in T_{\mathcal{K}}(y) \text { or } d \in \mathbb{R}_{+} \hat{y}, w \perp \hat{y}\right\} .
$$

Case 5: If $x \in \operatorname{bd} \mathcal{K} \backslash\{0\}$ and $y=0$, by symmetry to Case 4 , we have

$$
T_{\Omega}(x, y)=\left\{(d, w) \mid d \in T_{\mathcal{K}}(x), w=0 \text { or } d \perp \hat{x}, w \in \mathbb{R}_{+} \hat{x}\right\} .
$$

Case 6: If $x=0$ and $y=0$, then $\Pi_{\mathcal{K}}^{\prime}(0 ; h)=\Pi_{\mathcal{K}}(h)$ by Proposition 2.2 (iv). It follows from Proposition 5.2 that

$$
(d, w) \in T_{\Omega}(x, y) \Longleftrightarrow \Pi_{\mathcal{K}}(d-w)=d \quad \Longleftrightarrow \quad-w \in N_{\mathcal{K}}(d) \Longleftrightarrow(d, w) \in \Omega,
$$

i.e., $T_{\Omega}(x, y)=\Omega$. In fact, this case can also be obtained by noting that $\Omega$ is a cone.

When $m=1,2$, the tangent cone $T_{\Omega}$ have simpler expression given below. For example, when $m=1$, the second-order cone complementarity set $\Omega$ is reduced to the vector complementarity set $\left\{(a, b) \in \mathbb{R}^{2} \mid a \geq 0, b \geq 0, a b=0\right\}$, and hence bd $\mathcal{K} \backslash\{0\}$ is empty; when $m=2$, the condition $x_{1} \hat{w}-y_{1} d \in \mathbb{R} x$ can be dropped. 
Corollary 5.1 Let $\Omega$ be defined as in (16). If $m=1$, then

$$
T_{\Omega}(x, y)=\left\{\begin{array}{l|l}
(d, w) \mid \begin{array}{ll}
d=0, & \text { if } x=0, y>0 \\
w=0, & \text { if } x>0, y=0 \\
d \geq 0, w \geq 0, d \perp w, & \text { if } x=0, y=0
\end{array}
\end{array}\right\} .
$$

If $m=2$, then

$$
T_{\Omega}(x, y)=\left\{(d, w) \mid \begin{array}{ll}
d=0, w \in \mathbb{R}^{2}, & \text { if } x=0, y \in \operatorname{int} \mathcal{K} \\
d \in \mathbb{R}^{2}, w=0, & \text { if } x \in \operatorname{int} \mathcal{K}, y=0 \\
d \perp y, w \perp x, & \text { if } x, y \in \mathrm{bd} \mathcal{K} \backslash\{0\} \\
d=0, w \in T_{\mathcal{K}}(y) \text { or } d \in \mathbb{R}_{+} \hat{y}, w \perp \hat{y}, & \text { if } x=0, y \in \mathrm{bd} \mathcal{K} \backslash\{0\} \\
d \in T_{\mathcal{K}}(x), w=0 \text { or } d \perp \hat{x}, w \in \mathbb{R}_{+} \hat{x}, & \text { if } x \in \mathrm{bd} \mathcal{K} \backslash\{0\}, y=0 \\
d \in \mathcal{K}, w \in \mathcal{K}, d \perp w, & \text { if } x=0, y=0
\end{array}\right\} .
$$

Proof. If $m=1$, then $\mathcal{K}=\mathbb{R}_{+}$, and hence $\mathrm{bd} \mathcal{K} \backslash\{0\}=\emptyset$. Thus the desired result follows.

If $m=2$, we show that the condition $x_{1} \hat{w}-y_{1} d \in \mathbb{R} x$ is implied by $d \perp y$ and $w \perp x$ in the case of $x, y \in \mathrm{bd} \mathcal{K} \backslash\{0\}$ and $x^{T} y=0$. In fact, if $x_{1}=x_{2}>0$, then $y_{1}=-y_{2}$, and hence $w_{1}+w_{2}=0$ and $d_{1}-d_{2}=0$. Thus

$$
x_{1}\left[\begin{array}{c}
w_{1} \\
-w_{2}
\end{array}\right]-y_{1}\left[\begin{array}{l}
d_{1} \\
d_{2}
\end{array}\right]=x_{1}\left[\begin{array}{l}
w_{1} \\
w_{1}
\end{array}\right]-y_{1}\left[\begin{array}{l}
d_{1} \\
d_{1}
\end{array}\right]=\frac{x_{1} w_{1}-y_{1} d_{1}}{x_{1}}\left[\begin{array}{l}
x_{1} \\
x_{2}
\end{array}\right] .
$$

Similarly, if $x_{1}=-x_{2}$, then $y_{1}=y_{2}$, and hence $d_{1}+d_{2}=0$ and $w_{1}-w_{2}=0$. Thus

$$
x_{1}\left[\begin{array}{c}
w_{1} \\
-w_{2}
\end{array}\right]-y_{1}\left[\begin{array}{l}
d_{1} \\
d_{2}
\end{array}\right]=x_{1}\left[\begin{array}{c}
w_{1} \\
-w_{1}
\end{array}\right]-y_{1}\left[\begin{array}{c}
d_{1} \\
-d_{1}
\end{array}\right]=\frac{x_{1} w_{1}-y_{1} d_{1}}{x_{1}}\left[\begin{array}{c}
x_{1} \\
-x_{1}
\end{array}\right]=\frac{x_{1} w_{1}-y_{1} d_{1}}{x_{1}}\left[\begin{array}{l}
x_{1} \\
x_{2}
\end{array}\right] .
$$

\section{Expressions for directional normal cones}

In order to use FOSCMS for the second-order cone complementarity system, one needs to derive the exact formula for the directional normal cone of the second-order cone complementarity set. Moreover these results are of their own interest.

By formulating the vector complementarity set as the union of finitely many polyhedral convex sets, the formula of the directional normal cone of the vector complementarity set is given in [14, Lemma 4.1]. In contrast to the vector complementarity set, the second-order cone complementarity set cannot be represented as the union of finitely many polyhedral convex sets. In the following theorem we derive an explicit expression for the directional normal cone for the $m$-dimensional second-order cone complementarity set $\Omega$ defined as in (16). Note that in the case where $m=1, \mathrm{bd} \mathcal{K} \backslash\{0\}=\emptyset$ and hence the formula we derived reduced to the one given in [14, Lemma 4.1] for this case.

Theorem 6.1 The second-order cone complementarity set is directionally regular. For any $(x, y) \in \Omega$ and $(d, w) \in T_{\Omega}(x, y)=T_{\Omega}^{i}(x, y)$, the directional normal cone can be calculated as follows.

Case 1: $x=0, y \in \operatorname{int} \mathcal{K}$,

$$
N_{\Omega}((x, y) ;(d, w))=N_{\Omega}(x, y)=\mathbb{R}^{m} \times\{0\} .
$$


Case 2: $x \in \operatorname{int} \mathcal{K}, y=0$,

$$
N_{\Omega}((x, y) ;(d, w))=N_{\Omega}(x, y)=\{0\} \times \mathbb{R}^{m} .
$$

Case 3: $x, y \in \operatorname{bd} \mathcal{K} \backslash\{0\}$,

$$
N_{\Omega}((x, y) ;(d, w))=N_{\Omega}(x, y) .
$$

Case 4: $x \in \operatorname{bd} \mathcal{K} \backslash\{0\}, y=0$,

$$
N_{\Omega}((x, y) ;(d, w))=\left\{\begin{array}{l|l}
(u, v) \mid \begin{array}{ll}
u=0, v \in \mathbb{R}^{m} & \text { if } d \in \operatorname{int} T_{\mathcal{K}}(x), w=0 \\
N_{\Omega}(x, y) & \text { if } d \in \operatorname{bd} T_{\mathcal{K}}(x), w=0 \\
u \in \mathbb{R} \hat{x}, v \perp \hat{x} & \text { if } d \perp \hat{x}, w \in \mathbb{R}_{+} \hat{x} \backslash\{0\}
\end{array}
\end{array}\right\} .
$$

Case 5: $x=0, y \in \mathrm{bd} \mathcal{K} \backslash\{0\}$,

$$
N_{\Omega}((x, y) ;(d, w))=\left\{\begin{array}{l|l}
(u, v) \mid \begin{array}{ll}
u \in \mathbb{R}^{m}, v=0 & \text { if } d=0, w \in \operatorname{int} T_{\mathcal{K}}(x) \\
N_{\Omega}(x, y) & \text { if } d=0, w \in \operatorname{bd} T_{\mathcal{K}}(x) \\
v \in \mathbb{R} \hat{y}, u \perp \hat{y} & \text { if } d \in \mathbb{R}_{+} \hat{y} \backslash\{0\}, w \perp \hat{y}
\end{array}
\end{array}\right\} .
$$

Case 6: $x=0, y=0$,

$$
N_{\Omega}((x, y) ;(d, w))=N_{\Omega}(d, w) .
$$

Here the formula of the tangent cone and the normal cone of $\Omega$ are given as in Theorem 5.1 and Proposition 2.6 respectively.

Proof. In Cases 1-3, since it always has

$$
N_{\Omega}^{i}((x, y) ;(d, w)) \subseteq N_{\Omega}((x, y) ;(d, w)) \subseteq N_{\Omega}(x, y),
$$

it suffices to show that

$$
N_{\Omega}(x, y) \subseteq N_{\Omega}^{i}((x, y) ;(d, w)) .
$$

For any $(u, v) \in N_{\Omega}(x, y)$, in order to show that $(u, v) \in N_{\Omega}^{i}((x, y) ;(d, w))$, for any sequences $t_{n} \downarrow 0$, we need to find $\left(d^{n}, w^{n}\right) \rightarrow(d, w)$ and $\left(u^{n}, v^{n}\right) \rightarrow(u, v)$ satisfying $\left(u^{n}, v^{n}\right) \in$ $\widehat{N}_{\Omega}\left(x+t_{n} d^{n}, y+t_{n} w^{n}\right)$.

Case 1. $x=0, y \in \operatorname{int} \mathcal{K}$. Since $(u, v) \in N_{\Omega}(x, y),(d, w) \in T_{\Omega}(x, y)$, then by Theorem 5.1 , $d=0, w \in \mathbb{R}^{m}$ and by Proposition [2.6, $u \in \mathbb{R}^{m}, v=0$. By letting

$$
\left(u^{n}, v^{n}\right):=(u, v)=(u, 0) \text { and }\left(d^{n}, w^{n}\right):=(d, w)=(0, w),
$$

we have $y+t_{n} w^{n} \in \operatorname{int} \mathcal{K}$ for $n$ sufficiently large, and hence

$$
\left(u^{n}, v^{n}\right)=(u, 0) \in \widehat{N}_{\Omega}\left(0, y+t_{n} w\right)=\widehat{N}_{\Omega}\left(x+t_{n} d^{n}, y+t_{n} w^{n}\right) .
$$

Hence (19) holds.

Case 2. $x \in \operatorname{int} \mathcal{K}, y=0$. This case is symmetric to Case 1 and we omit the proof.

Case 3. $x, y \in \mathrm{bd} \mathcal{K} \backslash\{0\}$. Then $x-y \in(-\mathcal{K} \cup \mathcal{K})^{c}$. Since $(d, w) \in T_{\Omega}(x, y)=T_{\Omega}^{i}(x, y)$, by definition of the inner tangent cone, for any $t_{n} \downarrow 0$, there exists $\left(d^{n}, w^{n}\right) \rightarrow(d, w)$ such that $(x, y)+t_{n}\left(d^{n}, w^{n}\right) \in \Omega$. We now construct a sequence $\left(u^{n}, v^{n}\right)$ such that $\left(u^{n}, v^{n}\right) \rightarrow(u, v)$ and $\left(u^{n}, v^{n}\right) \in \widehat{N}_{\Omega}\left(x+t_{n} d^{n}, y+t_{n} w^{n}\right)$. By Proposition 2.1. $y=k \hat{x}$ with $k=y_{1} / x_{1}$. Hence $x_{1}-y_{1}=(1-k) x_{1}, x_{2}-y_{2}=(1+k) x_{2}$. By Proposition 2.3)(iii), the metric projection $\Pi_{\mathcal{K}}$ is differentiable at $x-y$ and

$$
\nabla \Pi_{\mathcal{K}}(x-y)=\frac{1}{2}\left[\begin{array}{cc}
1 & \bar{x}_{2}^{T} \\
\bar{x}_{2} & I+\frac{1-k}{1+k}\left(I-\bar{x}_{2} \bar{x}_{2}^{T}\right)
\end{array}\right]=\frac{1}{2}\left\{\left[\begin{array}{cc}
1 & \bar{x}_{2}^{T} \\
\bar{x}_{2}^{T} & I
\end{array}\right]+\frac{1-k}{1+k}\left[\begin{array}{cc}
0 & 0 \\
0 & I-\bar{x}_{2} \bar{x}_{2}^{T}
\end{array}\right]\right\} .
$$


By [4, Lemma 1], the eigenvalue values of the matrix are 0,1 and $1 / 1+k$ with multiplicity $n-2$.

Case 3(i): If $k \neq 1$, then the eigenvalue of the matrix $I-2 \nabla \Pi_{\mathcal{K}}(x-y)$ is $1,-1, \frac{k-1}{k+1}$.

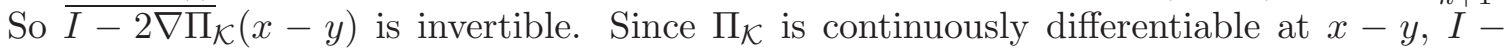
$2 \nabla \Pi_{\mathcal{K}}\left(x+t_{n} d^{n}-y-t_{n} w^{n}\right)$ is also invertible for sufficiently large $n$. Let

$\alpha(n):=\left(I-2 \nabla \Pi_{\mathcal{K}}\left(x+t_{n} d^{n}-y-t_{n} w^{n}\right)\right)^{-1}\left(\nabla \Pi_{\mathcal{K}}\left(x+t_{n} d^{n}-y-t_{n} w^{n}\right)-\nabla \Pi_{\mathcal{K}}(x-y)\right)(-u-v)$.

Then $\alpha(n) \rightarrow 0$ as $n \rightarrow \infty$. Note that

$$
\left(I-2 \nabla \Pi_{\mathcal{K}}\left(x+t_{n} d^{n}-y-t_{n} w^{n}\right)\right) \alpha(n)=\left(\nabla \Pi_{\mathcal{K}}\left(x+t_{n} d^{n}-y-t_{n} w^{n}\right)-\nabla \Pi_{\mathcal{K}}(x-y)\right)(-u-v)
$$

i.e.,

$$
\begin{aligned}
& \alpha(n) \\
= & \left(\nabla \Pi_{\mathcal{K}}\left(x+t_{n} d^{n}-y-t_{n} w^{n}\right)-\nabla \Pi_{\mathcal{K}}(x-y)\right)(-u-v)+2 \nabla \Pi_{\mathcal{K}}\left(x+t_{n} d^{n}-y-t_{n} w^{n}\right) \alpha(n) \\
= & \nabla \Pi_{\mathcal{K}}\left(x+t_{n} d^{n}-y-t_{n} w^{n}\right)(-u-v+2 \alpha(n))-\nabla \Pi_{\mathcal{K}}(x-y)(-u-v) \\
= & \nabla \Pi_{\mathcal{K}}\left(x+t_{n} d^{n}-y-t_{n} w^{n}\right)(-u-v+2 \alpha(n))-(-v),
\end{aligned}
$$

where the last step is due to $-v=\nabla \Pi_{\mathcal{K}}(x-y)(-u-v)$ since $(u, v) \in N_{\Omega}(x, y)$ by Proposition 2.4. Hence

$$
-v+\alpha(n)=\nabla \Pi_{\mathcal{K}}\left(x+t_{n} d^{n}-y-t_{n} w^{n}\right)(-u+\alpha(n)-v+\alpha(n)) .
$$

Let $\left(u^{n}, v^{n}\right):=(u-\alpha(n), v-\alpha(n))$. Then

$$
\left(u^{n}, v^{n}\right) \rightarrow(u, v) \text { and }-v^{n} \in \widehat{D}^{*} \Pi_{\mathcal{K}}\left(x+t_{n} d^{n}-y-t_{n} w^{n}\right)\left(-u^{n}-v^{n}\right) .
$$

By Proposition 2.4 $\left(u^{n}, v^{n}\right) \in \widehat{N}_{\Omega}\left(x+t_{n} d^{n}, y+t_{n} w^{n}\right)$. Hence (19) holds.

Case 3(ii): If $k=1$, then the eigenvalue of $I-2 \nabla \Pi_{\mathcal{K}}(x-y)$ is $1,-1, \frac{k-1}{k+1}=0$ and hence the matrix $I-2 \nabla \Pi_{\mathcal{K}}(x-y)$ is not invertible and the construction of $\left(u^{n}, v^{n}\right)$ in case 3(i) fails. Note that in this case the eigenvalue of the matrix $I-3 \nabla \Pi_{\mathcal{K}}(x-y)$ is $1,-2,-\frac{1}{2}$. So $I-3 \nabla \Pi_{\mathcal{K}}(x-y)$ has inverse. We then construct the sequence by taking $\left(u^{n}, v^{n}\right):=(u-2 \alpha(n), v-\alpha(n))$ with

$\alpha(n):=\left(I-3 \nabla \Pi_{\mathcal{K}}\left(x+t_{n} d^{n}-y-t_{n} w^{n}\right)\right)^{-1}\left(\nabla \Pi_{\mathcal{K}}\left(x+t_{n} d^{n}-y-t_{n} w^{n}\right)-\nabla \Pi_{\mathcal{K}}(x-y)\right)(-u-v)$,

and the desired result follows similarly.

Case 4. $x \in \mathrm{bd} \mathcal{K} \backslash\{0\}$ and $y=0$. Since $(d, w) \in T_{\Omega}(x, y)$, by Theorem 5.1, there are three possible cases: $w=0, d \in \operatorname{int} T_{\mathcal{K}}(x), w=0, d \in \operatorname{bd} T_{\mathcal{K}}(x)$, or $d \perp \hat{x}, w \in \mathbb{R}_{+} \hat{x} \backslash\{0\}$.

$\underline{\text { Subcase 4.1. }} w=0$ and $d \in \operatorname{int} T_{\mathcal{K}}(x)$. Since $(d, w) \in T_{\Omega}(x, y)=T_{\Omega}^{i}(x, y)$, then for any $t_{n} \downarrow 0$, there exists $\left(d^{n}, w^{n}\right) \rightarrow(d, w)$ such that $(x, y)+t_{n}\left(d^{n}, w^{n}\right) \in \Omega$. In this case $\bar{x}_{2}^{T} d_{2}-d_{1}<0$. Hence $\left\|x_{2}+t_{n} d_{2}^{n}\right\|=\left\|x_{2}\right\|+t_{n} \bar{x}_{2}^{T} d_{2}^{n}+o\left(t_{n}\right)<x_{1}+t_{n} d_{1}^{n}$ for sufficiently large $n$. So $x+t_{n} d^{n} \in \operatorname{int} \mathcal{K}$. It follows that

$$
\widehat{N}_{\Omega}\left(x+t_{n} d^{n}, t_{n} w^{n}\right)=\left\{(u, v) \mid u=0, v \in \mathbb{R}^{m}\right\} .
$$


Hence

$$
N_{\Omega}((x, y) ;(d, 0))=N_{\Omega}^{i}((x, y) ;(d, 0))=\left\{(u, v) \mid u=0, v \in \mathbb{R}^{m}\right\} .
$$

$\underline{\text { Subcase 4.2. }} w=0$ and $d \in \operatorname{bd} T_{\mathcal{K}}(x)$. In this case, it suffices to show

$$
N_{\Omega}(x, y) \subseteq N_{\Omega}^{i}((x, y) ;(d, w)) .
$$

By Proposition [2.6. for any $(u, v) \in N_{\Omega}(x, y)$, there are three possible cases: $u=0, v \in \mathbb{R}^{m}$ or $u \in \mathbb{R} \hat{x}, v \perp \hat{x}$ or $u \in \mathbb{R}_{-} \hat{x},\langle v, \hat{x}\rangle \leq 0$.

Subcase 4.2(i). $u=0$ and $v \in \mathbb{R}^{m}$. Since $d \in \operatorname{bd} T_{\mathcal{K}}(x)$, we have $x_{1} d_{1}-x_{2}^{T} d_{2}=0$ by the formula for the tangent cone. For $t_{n} \downarrow 0$, let $\eta\left(t_{n}\right):=\left\|x_{2}+t_{n} d_{2}\right\|-\left\|x_{2}\right\|-t_{n} \bar{x}_{2}^{T} d_{2}, w^{n}:=0$, and

$$
d^{n}:= \begin{cases}\left(d_{1}+2\left|\eta\left(t_{n}\right)\right| / t_{n}, d_{2}\right), & \text { if } \eta\left(t_{n}\right) \neq 0 \\ \left(d_{1}+t_{n}, d_{2}\right), & \text { otherwise }\end{cases}
$$

If $\eta\left(t_{n}\right) \neq 0$, then

$$
x_{1}+t_{n} d_{1}^{n}=x_{1}+t_{n} d_{1}+2\left|\eta\left(t_{n}\right)\right|>\left\|x_{2}\right\|+t_{n} \bar{x}_{2}^{T} d_{2}+\eta\left(t_{n}\right)=\left\|x_{2}+t_{n} d_{2}^{n}\right\| ;
$$

otherwise

$$
x_{1}+t_{n} d_{1}^{n}=x_{1}+t_{n} d_{1}+t_{n}^{2}=\left\|x_{2}\right\|+t_{n} \bar{x}_{2}^{T} d_{2}+t_{n}^{2}>\left\|x_{2}+t_{n} d_{2}\right\| .
$$

Hence $x+t_{n} d^{n} \in \operatorname{int} \mathcal{K}$ and $y+t_{n} w^{n}=0$. This ensures $(u, v) \in \widehat{N}_{\Omega}\left(x+t_{n} d^{n}, 0\right)=$ $\widehat{N}_{\Omega}\left(x+t_{n} d^{n}, y+t_{n} w^{n}\right)$. So $(u, v) \in N_{\Omega}^{i}((x, y) ;(d, w))$.

Subcase 4.2(ii). $u \in \mathbb{R} \hat{x}$ and $v \perp \hat{x}$. In this case, $u_{2}=-u_{1} \bar{x}_{2}$ and $v_{1}-\bar{x}_{2}^{T} v_{2}=0$. This is equivalent to

$$
\begin{aligned}
\left\{\begin{array}{l}
u_{1}=v_{1}-\bar{x}_{2}^{T}\left(u_{2}+v_{2}\right) \\
u_{2}=-u_{1} \bar{x}_{2}
\end{array}\right. & \Longleftrightarrow\left\{\begin{array}{l}
u_{1}=v_{1}-\bar{x}_{2}^{T}\left(u_{2}+v_{2}\right) \\
2 u_{2}=-\left(u_{1}+v_{1}\right) \bar{x}_{2}+\left(v_{1}-u_{1}\right) \bar{x}_{2}
\end{array}\right. \\
& \Longleftrightarrow\left\{\begin{array}{l}
u_{1}=v_{1}-\bar{x}_{2}^{T}\left(u_{2}+v_{2}\right) \\
2 u_{2}=-\left(u_{1}+v_{1}\right) \bar{x}_{2}+\left(u_{2}+v_{2}\right)^{T} \bar{x}_{2} \bar{x}_{2}
\end{array}\right. \\
& \Longleftrightarrow 2 u=\left[\begin{array}{cc}
1 & -\bar{x}_{2}^{T} \\
-\bar{x}_{2} & \bar{x}_{2} \bar{x}_{2}^{T}
\end{array}\right](u+v) \\
& \Longleftrightarrow v=\left(I+\frac{1}{2}\left[\begin{array}{cc}
-1 & \bar{x}_{2}^{T} \\
\bar{x}_{2} & -\bar{x}_{2} \bar{x}_{2}^{T}
\end{array}\right]\right)(u+v) .
\end{aligned}
$$

The following argument is similar to Case 3. Let

$$
M:=I+\frac{1}{2}\left[\begin{array}{cc}
-1 & \bar{x}_{2}^{T} \\
\bar{x}_{2} & -\bar{x}_{2} \bar{x}_{2}^{T}
\end{array}\right] .
$$

First note that $I-2 M=-I+\left[\begin{array}{cc}1 & -\bar{x}_{2}^{T} \\ -\bar{x}_{2} & \bar{x}_{2} \bar{x}_{2}^{T}\end{array}\right]$ has the eigenvalue 1 and -1 with multiplicity $n-1$. Hence it is invertible. For any $t_{n} \downarrow 0$, take $d^{n}=\left(d_{1}^{n}, d_{2}^{n}\right)$ with

$$
d_{1}^{n}:=\frac{\left\|x_{2}+t_{n} d_{2}\right\|-\left\|x_{2}\right\|}{t_{n}}, d_{2}^{n}:=d_{2}, \text { and } w^{n}:=t_{n}\left(\hat{x}+t_{n} \widehat{d^{n}}\right) .
$$


So $d^{n} \rightarrow d$ and $w^{n} \rightarrow 0$. Then $\left(x+t_{n} d^{n}, t_{n} w^{n}\right) \in \Omega$ with $x+t_{n} d^{n}, t_{n} w^{n} \in \operatorname{bd} \mathcal{K} \backslash\{0\}$. Let $z^{n}:=x+t_{n} d^{n}-t_{n} w^{n}$. Then $z^{n} \rightarrow x$ and

$$
\nabla \Pi_{\mathcal{K}}\left(z^{n}\right)=I+\frac{1}{2}\left[\begin{array}{cc}
-1 & \frac{\left(z_{2}^{n}\right)^{T}}{\left\|z_{2}^{n}\right\|} \\
\frac{z_{2}^{n}}{\left\|z_{2}^{n}\right\|} & -I+\frac{z_{1}^{n}}{\left\|z_{2}^{n}\right\|}\left(I-\frac{z_{2}^{n}}{\left\|z_{2}^{n}\right\|} \frac{\left(z_{2}^{n}\right)^{T}}{\left\|z_{2}^{n}\right\|}\right)
\end{array}\right] \rightarrow I+\frac{1}{2}\left[\begin{array}{cc}
-1 & \bar{x}_{2}^{T} \\
\bar{x}_{2} & -\bar{x}_{2} \bar{x}_{2}^{T}
\end{array}\right]=M
$$

Hence $I-2 \nabla \Pi_{\mathcal{K}}\left(x+t_{n} d^{n}-t_{n} w^{n}\right)$ is inverse as $n$ is large enough. Let

$$
\alpha(n):=\left(I-2 \nabla \Pi_{\mathcal{K}}\left(x+t_{n} d^{n}-t_{n} w^{n}\right)\right)^{-1}\left(\nabla \Pi_{\mathcal{K}}\left(x+t_{n} d^{n}-t_{n} w^{n}\right)-M\right)(-u-v) .
$$

Then

$$
\left(I-2 \nabla \Pi_{\mathcal{K}}\left(x+t_{n} d^{n}-t_{n} w^{n}\right)\right) \alpha(n)=\left(\nabla \Pi_{\mathcal{K}}\left(x+t_{n} d^{n}-t_{n} w^{n}\right)-M\right)(-u-v) .
$$

Hence

$$
\begin{aligned}
\alpha(n) & =\left(\nabla \Pi_{\mathcal{K}}\left(x+t_{n} d^{n}-t_{n} w^{n}\right)-M\right)(-u-v)+2 \nabla \Pi_{\mathcal{K}}\left(x+t_{n} d^{n}-t_{n} w^{n}\right) \alpha(n) \\
& =\nabla \Pi_{\mathcal{K}}\left(x+t_{n} d^{n}-t_{n} w^{n}\right)(-u+\alpha(n)-v+\alpha(n))-M(-u-v) .
\end{aligned}
$$

This together with $v=M(u+v)$ yields

$$
-v+\alpha(n)=D^{*} \Pi_{\mathcal{K}}\left(x+t_{n} d^{n}-t_{n} w^{n}\right)(-u+\alpha(n)-v+\alpha(n)) .
$$

So $(u-\alpha(n), v-\alpha(n)) \in \widehat{N}_{\Omega}\left(x+t_{n} d^{n}, y+t_{n} w^{n}\right)$. Thus $(u, v) \in N_{\Omega}^{i}((x, y) ;(d, w))$.

Subcase 4.2(iii). $u \in \mathbb{R}_{-} \hat{x}$ and $\langle v, \hat{x}\rangle \leq 0$. For any $t_{n} \downarrow 0$, let $w^{n}:=0$ and $d^{n}=\left(d_{1}^{n}, d_{2}^{n}\right)$ with $d_{1}^{n}:=\frac{\left\|x_{2}+t_{n} d_{2}\right\|-x_{1}}{t_{n}}$ and $d_{2}^{n}:=d_{2}$. Then $d^{n} \rightarrow d, w^{n} \rightarrow w$, and $x+t_{n} d^{n} \in \operatorname{bd} \mathcal{K} \backslash\{0\}$. Let $z^{n}:=\hat{x}+t_{n} \widehat{d^{n}}$ and

$$
u^{n}:=\frac{u_{1}}{x_{1}} z^{n} \text { and } v^{n}:=v-t_{n} \frac{\left\langle\hat{v}, d^{n}\right\rangle}{\left\|z^{n}\right\|} \frac{z^{n}}{\left\|z^{n}\right\|} .
$$

Then $v^{n} \rightarrow v$ and $u^{n} \rightarrow \frac{u_{1}}{x_{1}} \hat{x}=u$, where $\frac{u_{1}}{x_{1}} \leq 0$ and $u=\frac{u_{1}}{x_{1}} \hat{x}$ is due to $u \in \mathbb{R}_{-} \hat{x}$. Note that $u^{n} \in \mathbb{R}_{-} z^{n}$ and

$$
\left\langle v^{n}, z^{n}\right\rangle=\left\langle v, z^{n}\right\rangle-t_{n}\left\langle\hat{v}, d^{n}\right\rangle=\left\langle\hat{v}, x+t_{n} d^{n}\right\rangle-t_{n}\left\langle\hat{v}, d^{n}\right\rangle=\langle v, \hat{x}\rangle \leq 0 .
$$

This means $\left(u^{n}, v^{n}\right) \in \widehat{N}_{\Omega}\left(\widehat{z^{n}}, 0\right)=\widehat{N}_{\Omega}\left(x+t_{n} d^{n}, y+t_{n} w^{n}\right)$. So $(u, v) \in N_{\Omega}^{i}((x, y) ;(d, w))$.

Subcase 4.3. $d \perp \hat{x}$ and $w \in \mathbb{R}_{+} \hat{x} \backslash\{0\}$. In this case, we will show that

$$
N_{\Omega}^{i}((x, y) ;(d, w))=N_{\Omega}((x, y) ;(d, w))=\{(u, v) \mid u \in \mathbb{R} \hat{x}, v \perp \hat{x}\} .
$$

Take $(u, v) \in N_{\Omega}((x, y) ;(d, w))$. Then there exist sequences $t_{n} \downarrow 0,\left(d^{n}, w^{n}\right) \rightarrow(d, w),\left(u^{n}, v^{n}\right) \rightarrow$ $(u, v)$ such that $\left(u^{n}, v^{n}\right) \in \widehat{N}_{\Omega}\left(x+t_{n} d^{n}, t_{n} w^{n}\right)$. Since $x \in \mathrm{bd} \mathcal{K} \backslash\{0\}$ and $w \neq 0$, for $n$ sufficiently large, $0 \neq x+t_{n} d^{n} \in \mathcal{K}$ and $0 \neq t_{n} w^{n}$. It follows that $x+t_{n} d^{n}, t_{n} w^{n} \in \operatorname{bd} \mathcal{K} \backslash\{0\}$. Hence, by Proposition 2.6.

$$
u^{n} \perp x+t_{n} d^{n}, \quad v^{n} \perp t_{n} w^{n}, \quad\left(x+t_{n} d^{n}\right)_{1} \widehat{u^{n}}+\left(t_{n} w^{n}\right)_{1} v^{n} \in \mathbb{R}\left[x+t_{n} d^{n}\right] .
$$


Taking the limits yields $u \perp x, v \perp w, \hat{u} \in \mathbb{R} x$, which together with $w \in \mathbb{R}_{+} \hat{x} \backslash\{0\}$ implies that $v \perp \hat{x}, u \in \mathbb{R} \hat{x}$. Hence

$$
N_{\Omega}((x, y) ;(d, w)) \subseteq\{(u, v) \mid u \in \mathbb{R} \hat{x}, v \perp \hat{x}\} .
$$

Conversely, let $u \in \mathbb{R} \hat{x}$ and $v \perp \hat{x}$. Similarly to Subcase 4.2 (ii), we can prove $(u, v) \in$ $N_{\Omega}^{i}((x, y) ;(d, w))$. The only change is to take $w^{n}:=\frac{w_{1}}{x_{1}}\left(\hat{x}+t_{n} \widehat{d^{n}}\right)$ instead of $w^{n}=t_{n}(\hat{x}+$

$\left.t_{n} \widehat{d^{n}}\right)$. Since $w=\tau \hat{x}$ for some $\tau>0$, we have $w_{2}=\frac{w_{1}}{x_{1}}\left(-x_{2}\right)$, and hence $w^{n}=\frac{w_{1}}{x_{1}}\left(\hat{x}+t_{n} \widehat{d^{n}}\right) \rightarrow$ $\frac{w_{1}}{x_{1}} \hat{x}=w$.

Case 5. $x=0$ and $y \in \mathrm{bd} \mathcal{K} /\{0\}$. The result follows by a symmetric analysis of Case 4 .

Case 6. If $x=0$ and $y=0$, then $(d, w) \in T_{\Omega}(x, y)=\Omega$. Using Proposition 3.4 yields

$$
N_{\Omega}^{i}((x, y) ;(d, w))=N_{\Omega}((x, y) ;(d, w))=N_{\Omega}(d, w) .
$$

\section{Sufficient conditions for error bounds of the second-order cone complementarity system}

In this section, we give verifiable sufficient conditions for the error bound property of the second-order cone complementarity system (11)-(2). First by applying Theorem 4.1 we have the following sufficient conditions based on the limiting normal cones.

Theorem 7.1 Given a point $z^{*} \in \mathcal{F}$. The system (11)-(2) has a local error bound at $z^{*}$ if the NNAMCQ holds at $z^{*}$ :

$$
\left.\begin{array}{l}
\nabla F\left(z^{*}\right)^{T} \lambda^{F}+\sum_{i=1}^{J}\left\{\nabla G_{i}\left(z^{*}\right)^{T} \lambda_{i}^{G}+\nabla H_{i}\left(z^{*}\right)^{T} \lambda_{i}^{H}\right\}=0, \\
\lambda^{F} \in N_{\Lambda}\left(F\left(z^{*}\right)\right), \quad\left(\lambda^{G}, \lambda^{H}\right) \in N_{\Omega}\left(G\left(z^{*}\right), H\left(z^{*}\right)\right)
\end{array}\right\} \Longrightarrow\left(\lambda^{F}, \lambda^{G}, \lambda^{H}\right)=0 .
$$

Here the exact expression for the limiting normal cone of $\Omega$ can be found in Proposition 2.6.

By applying Theorems 4.2 and 4.4 respectively, we obtain the sufficient conditions in Theorems 7.2 and 7.3 based on directional limiting normal cone immediately. According to the relationship between the limiting normal cone and the directional limiting normal cone $N_{C}(z ; d) \subseteq N_{C}(z)$, the sufficient condition based on the directional limiting normal cone is in general weaker than the one based on the limiting normal cone given in Theorem 7.1 . In fact Example 4.1 shows that it is possible that the NNAMCQ does not hold while the sufficient condition in terms of the directional limiting normal cone holds. Note that in the following theorem, the formula of the tangent cone and the directional normal cone for a second-order cone complementarity set can be found in Theorems 5.1 and 6.1, respectively. Moreover, the equivalence of the two conditions are due to the directional regularity of the second-order cone complementarity set proved in Theorem 6.1.

Theorem 7.2 Given a point $z^{*} \in \mathcal{F}$. Suppose that for every $0 \neq w \in \mathbb{R}^{n}$ with $\nabla F\left(z^{*}\right) w \in$ $T_{\Lambda}\left(F\left(z^{*}\right)\right),\left(\nabla G_{i}\left(z^{*}\right) w, \nabla H_{i}\left(z^{*}\right) w\right) \in T_{\Omega_{i}}\left(G_{i}\left(z^{*}\right), H_{i}\left(z^{*}\right)\right), i=1, \ldots, J$, one has

$$
\left.\begin{array}{l}
\nabla F\left(z^{*}\right)^{T} \lambda^{F}+\sum_{i=1}^{J}\left\{\nabla G_{i}\left(z^{*}\right)^{T} \lambda_{i}^{G}+\nabla H_{i}\left(z^{*}\right)^{T} \lambda_{i}^{H}\right\}=0, \\
\lambda^{F} \in N_{\Lambda}\left(F\left(z^{*}\right) ; \nabla F\left(z^{*}\right) w\right), \\
\left(\lambda_{i}^{G}, \lambda_{i}^{H}\right) \in N_{\Omega_{i}}\left(\left(G_{i}\left(z^{*}\right), H_{i}\left(z^{*}\right)\right) ;\left(\nabla G_{i}\left(z^{*}\right) w, \nabla H_{i}\left(z^{*}\right) w\right)\right), i=1, \ldots, J
\end{array}\right\} \Longrightarrow\left(\lambda^{F}, \lambda^{G}, \lambda^{H}\right)=0,
$$


or equivalently for every $0 \neq w \in \mathbb{R}^{n}$ with $\nabla F\left(z^{*}\right) w \in T_{\Lambda}\left(F\left(z^{*}\right)\right),\left(\nabla G\left(z^{*}\right) w, \nabla H\left(z^{*}\right) w\right) \in$ $T_{\Omega}\left(G\left(z^{*}\right), H\left(z^{*}\right)\right)$ one has

$$
\left.\begin{array}{l}
\nabla F\left(z^{*}\right)^{T} \lambda^{F}+\nabla G\left(z^{*}\right)^{T} \lambda^{G}+\nabla H\left(z^{*}\right)^{T} \lambda^{H}=0, \\
\lambda^{F} \in N_{\Lambda}\left(F\left(z^{*}\right) ; \nabla F\left(z^{*}\right) w\right), \\
\left(\lambda^{G}, \lambda^{H}\right) \in N_{\Omega}\left(\left(G\left(z^{*}\right), H\left(z^{*}\right)\right) ;\left(\nabla G\left(z^{*}\right) w, \nabla H\left(z^{*}\right) w\right)\right)
\end{array}\right\} \Longrightarrow\left(\lambda^{F}, \lambda^{G}, \lambda^{H}\right)=0 .
$$

Then the system (1)-(2) has a local error bound at $z^{*}$.

Theorem 7.3 Given a point $z^{*} \in \mathcal{F}$. Suppose that the set-valued mapping $M(z):=F(z)-$ $\Lambda$ is metrically subregular at $\left(z^{*}, 0\right)$. Further assume that for every $0 \neq w \in \mathbb{R}^{n}$ with $\nabla F\left(z^{*}\right) w \in T_{\Lambda}\left(F\left(z^{*}\right)\right),\left(\nabla G_{i}\left(z^{*}\right) w, \nabla H_{i}\left(z^{*}\right) w\right) \in T_{\Omega_{i}}\left(G_{i}\left(z^{*}\right), H_{i}\left(z^{*}\right)\right), i=1, \ldots, J$, one has

$$
\left.\begin{array}{l}
\nabla F\left(z^{*}\right)^{T} \lambda^{F}+\sum_{i=1}^{J}\left\{\nabla G_{i}\left(z^{*}\right)^{T} \lambda_{i}^{G}+\nabla H_{i}\left(z^{*}\right)^{T} \lambda_{i}^{H}\right\}=0, \\
\lambda^{F} \in N_{\Lambda}\left(F\left(z^{*}\right) ; \nabla F\left(z^{*}\right) w\right), \\
\left(\lambda_{i}^{G}, \lambda_{i}^{H}\right) \in N_{\Omega_{i}}\left(\left(G_{i}\left(z^{*}\right), H_{i}\left(z^{*}\right)\right) ;\left(\nabla G_{i}\left(z^{*}\right) w, \nabla H_{i}\left(z^{*}\right) w\right)\right), i=1, \ldots, J
\end{array}\right\} \Longrightarrow\left(\lambda^{G}, \lambda^{H}\right)=0,
$$

or equivalently for every $0 \neq w \in \mathbb{R}^{n}$ with $\nabla F\left(z^{*}\right) w \in T_{\Lambda}\left(F\left(z^{*}\right)\right),\left(\nabla G\left(z^{*}\right) w, \nabla H\left(z^{*}\right) w\right) \in$ $T_{\Omega}\left(G\left(z^{*}\right), H\left(z^{*}\right)\right)$ one has

$$
\left.\begin{array}{l}
\nabla F\left(z^{*}\right)^{T} \lambda^{F}+\nabla G\left(z^{*}\right)^{T} \lambda^{G}+\nabla H\left(z^{*}\right)^{T} \lambda^{H}=0, \\
\lambda^{F} \in N_{\Lambda}\left(F\left(z^{*}\right) ; \nabla F\left(z^{*}\right) w\right), \\
\left(\lambda^{G}, \lambda^{H}\right) \in N_{\Omega}\left(\left(G\left(z^{*}\right), H\left(z^{*}\right)\right) ;\left(\nabla G\left(z^{*}\right) w, \nabla H\left(z^{*}\right) w\right)\right)
\end{array}\right\} \Longrightarrow\left(\lambda^{G}, \lambda^{H}\right)=0 .
$$

Then the system (1)-(2) has a local error bound at $z^{*}$.

In order to use Theorem 7.3 , the set-valued mapping $M(z):=F(z)-\Lambda$ should satisfy the metric subregularity. For convenience, we summarize some prominent sufficient conditions for the case of an equality and inequality system in the following theorem. It is well known that in Theorem 7.4 (ii) $\Longrightarrow$ (iii) $\Longleftrightarrow$ (iv) $\Longrightarrow$ (v) or (vi), (i) $\Longrightarrow$ (v) and (i) $\Longrightarrow$ (vi).

Theorem 7.4 (Sufficient conditions for MS for the equality and inequality system) Let $z^{*}$ be a feasible point to the system $g(z) \leq 0, h(z)=0$, where $g: \mathbb{R}^{n} \rightarrow \mathbb{R}^{p}, h: \mathbb{R}^{n} \rightarrow \mathbb{R}^{q}$ are differentiable. Then the set-valued mapping $M(z):=(g(z), h(z))-\mathbb{R}_{-}^{p} \times\{0\}^{q}$ is metrically subregular at $\left(z^{*}, 0\right)$ under one of the following conditions.

(i) Linearity constraint qualification (Linear CQ) holds: h, g are affine.

(ii) Linear independence constraint qualification (LICQ) holds: $\left\{\nabla g_{i}\left(z^{*}\right), \nabla h_{j}\left(z^{*}\right) \mid i \in\right.$ $\left.I_{g}, j=1, \ldots, q\right\}$ are linearly independent.

(iii) The Mangasarian-Fromovitz constraint qualification (MFCQ) holds at $z^{*}:\left\{\nabla h_{i}\left(z^{*}\right) \mid i=\right.$ $1, \ldots, q\}$ are linearly independent and there exists $d \in \mathbb{R}^{n}$ such that $\nabla h_{i}\left(z^{*}\right) d=0$ for all $i=1, \ldots, q$ and $\nabla g_{i}\left(z^{*}\right) d<0$ for all $i \in I_{g}$.

(iv) NNAMCQ holds at $z^{*}$ :

$$
\begin{aligned}
& \nabla g\left(z^{*}\right)^{T} \lambda^{g}+\nabla h\left(z^{*}\right)^{T} \lambda^{h}=0, \quad \lambda^{g} \geq 0,\left\langle\lambda^{g}, g\left(z^{*}\right)\right\rangle=0 \\
& \Longrightarrow\left(\lambda^{g}, \lambda^{h}\right)=0 .
\end{aligned}
$$


(v) Quasinormality holds at $z^{*}([17$, Corollary 5.3]):

$$
\begin{aligned}
& \left\{\begin{array}{l}
(20) \text { and there exists a sequence }\left\{z^{k}\right\} \text { converging to } z^{*} \text { such that for each } k, \\
\lambda_{i}^{g}>0 \Longrightarrow g_{i}\left(z^{k}\right)>0, \lambda_{i}^{h} \neq 0 \Longrightarrow \lambda_{i}^{h} h_{i}\left(z^{k}\right)>0
\end{array}\right. \\
& \Longrightarrow\left(\lambda^{g}, \lambda^{h}\right)=0 .
\end{aligned}
$$

(vi) The relaxed constant positive linear dependence condition $(R C P L D)$ holds at $z^{*}(\sqrt{18}$, Theorem 4.2]): Let $\mathcal{J} \subseteq\{1, \ldots, q\}$ and $\left\{\nabla h_{j}\left(z^{*}\right) \mid j \in \mathcal{J}\right\}$ be a basis for $\operatorname{span}\left\{\nabla h_{j}\left(z^{*}\right) \mid j=\right.$ $1, \ldots, q\}$. There exists $\delta>0$ such that

- $\left\{\nabla h_{j}(z)\right\}_{j=1}^{q}$ has the same rank for each $z \in B_{\delta}\left(z^{*}\right)$;

- for each $\mathcal{I} \subseteq I_{g}$, if $\left\{\nabla g_{i}\left(z^{*}\right), \nabla h_{j}\left(z^{*}\right) \mid i \in \mathcal{I}, j \in \mathcal{J}\right\}$ is positively linear dependent, then $\left\{\nabla g_{i}(z), \nabla h_{j}(z) \mid i \in \mathcal{I}, j \in \mathcal{J}\right\}$ is linear dependent for each $z \in B_{\delta}\left(z^{*}\right)$.

(vii) There are no nonzero direction in the linearized cone ([15, Corollary 1]):

$$
\nabla g_{i}\left(z^{*}\right) d \leq 0, i \in I_{g}, \nabla h_{i}\left(z^{*}\right) d=0, i=1, \ldots, q \Longrightarrow d=0 .
$$

(viii) Second-order sufficient condition for metric subregularity (SOSCMS) (15, Corollary 1]): For every $0 \neq w \in \mathbb{R}^{n}$ with $\nabla g_{i}\left(z^{*}\right) w \leq 0$ for $i \in I_{g}$ and $\nabla h_{i}\left(z^{*}\right) w=0$ for $i=1, \ldots, q$, one has

$$
\left.\begin{array}{l}
\nabla g\left(z^{*}\right)^{T} \lambda^{g}+\nabla h\left(z^{*}\right)^{T} \lambda^{h}=0, \quad \lambda^{g} \geq 0,\left\langle\lambda^{g}, g\left(z^{*}\right)\right\rangle=0 \\
w^{T} \nabla^{2}\left(\left(\lambda^{g}\right)^{T} g\right)\left(z^{*}\right) w+w^{T} \nabla^{2}\left(\left(\lambda^{h}\right)^{T} h\right)\left(z^{*}\right) w \geq 0
\end{array}\right\} \Longrightarrow\left(\lambda^{g}, \lambda^{h}\right)=0 .
$$

The following example shows that if there exists $0 \neq w \in \mathbb{R}^{n}$ with $\nabla g_{i}\left(z^{*}\right) w \leq 0$ for $i \in I_{g}$ and $\nabla h_{i}\left(z^{*}\right) w=0$ for $i=1, \ldots, q$, then SOSCMS is weaker than NNAMCQ, or equivalently MFCQ.

Example 7.1 Let $g_{1}(z)=z_{1}-z_{2}^{2}, g_{2}(z)=z_{1}^{2}-z_{2}$, and $h(z)=z_{1}$. At $z=(0,0)$, consider

$$
\lambda^{g_{1}} \nabla g_{1}(z)+\lambda^{g_{2}} \nabla g_{2}(z)+\lambda^{h} \nabla h(z)=0
$$

with $\lambda^{g_{1}}, \lambda^{g_{2}} \geq 0$. Then we can take $\left(\lambda^{g_{1}}, \lambda^{g_{2}}, \lambda^{h}\right)=(1,0,-1) \neq(0,0,0)$. So MFCQ fails at $z=(0,0)$. Let $w$ satisfying $\nabla h(z) w=0$. Then $w_{1}=0$, and hence from

$$
\lambda^{g_{1}} w^{T} \nabla^{2} g_{1}(z) w+\lambda^{g_{2}} w^{T} \nabla^{2} g_{2}(z) w \geq 0
$$

we have $-2 \lambda^{g_{1}} w_{2}^{2} \geq 0$, so $\lambda^{g_{1}} \leq 0$ and hence $\lambda^{g_{1}}=0$. Consequently, $\lambda^{g_{2}}=0$ and $\lambda^{h}=0$. So SOSCMS holds at $z=(0,0)$.

Our sufficient condition Theorem 7.3 provides a sufficient condition for metric subregularity for the very general system (11)-(2). There may exist more than one way to split a system and this provides flexibility in using Theorem 7.3. For example, suppose that a second-order cone complementarity system consists only (11). Suppose some of the subsystems, without loss of generality,

$$
\left(G_{i}(z), H_{i}(z)\right) \in \Omega_{i}, \quad i=1, \ldots, s,
$$


where $s \leq J$ is metric subregular at $\left(z^{*}, 0\right)$. Then one can split the original system (10) as

$$
\begin{aligned}
& \mathcal{K}_{i} \ni G_{i}(z) \perp H_{i}(z) \in \mathcal{K}_{i} \quad i=s+1, \ldots, J, \\
& F(z) \in \Lambda,
\end{aligned}
$$

where

$$
F(z):=\left(G_{1}(z), H_{1}(z)\right) \times \cdots \times\left(G_{s}(z), H_{s}(z)\right) \text { and } \Lambda:=\Omega_{1} \times \cdots \times \Omega_{s},
$$

and use Theorem 7.3. In particular, since $\mathcal{K}_{i}$ with $m_{i}=1$ is the set of nonnegative reals $\mathbb{R}_{+}, \Omega_{i}$ with $m_{i}=1$ is equal to the vector complementarity cone $\Theta:=\left\{(x, y) \in \mathbb{R}^{2} \mid x \geq\right.$ $\left.0, y \geq 0, x^{T} y=0\right\}$. Without loss of generality, assuming $m_{i}=1$ for $i=1, \ldots, s$, then the system $F(z) \in \Lambda$ given in (21) is then equal to the vector complementarity system

$$
F(z) \in \Theta^{s}
$$

where $\Theta^{s}:=\left\{(a, b) \in \mathbb{R}^{2 s} \mid a \geq 0, b \geq 0, a^{T} b=0\right\}$. We now summarize some prominent sufficient conditions for metric subregularity for the vector complementarity system in the following theorem.

Theorem 7.5 (Sufficient conditions for MS for a complementarity system) Let $z^{*}$ be a feasible point to the vector complementarity system $g(z) \leq 0, h(z)=0,0 \leq \phi(z) \perp$ $\psi(z) \geq 0$, where $g: \mathbb{R}^{n} \rightarrow \mathbb{R}^{p}, h: \mathbb{R}^{n} \rightarrow \mathbb{R}^{q}, \phi: \mathbb{R}^{n} \rightarrow \mathbb{R}^{s}, \psi: \mathbb{R}^{n} \rightarrow \mathbb{R}^{s}$ are continuously differentiable. Then, the set-valued mapping $M(z):=(g(z), h(z), \phi(z), \psi(z))-\mathbb{R}_{-}^{p} \times\{0\}^{q} \times \Theta^{s}$ is metrically subregular at $\left(z^{*}, 0\right)$ under one of the following conditions:

(i) Linearity $C Q$ holds: $g, h, \phi, \psi$ are affine.

(ii) MPEC LICQ holds: $\left\{\nabla g_{i}\left(z^{*}\right)\left(i \in I_{g}\right), \nabla h_{i}\left(z^{*}\right)(i=1, \ldots, q), \nabla \phi_{i}\left(z^{*}\right)\left(i \in I_{\phi}\right), \nabla \psi_{i}\left(z^{*}\right)(i \in\right.$ $\left.\left.I_{\psi}\right)\right\}$ are linearly independent.

(iii) MPEC NNAMCQ holds at $z^{*}$ :

$$
\begin{aligned}
& \nabla g\left(z^{*}\right)^{T} \lambda^{g}+\nabla h\left(z^{*}\right)^{T} \lambda^{h}+\nabla \phi\left(z^{*}\right)^{T} \lambda^{\phi}+\nabla \psi\left(z^{*}\right)^{T} \lambda^{\psi}=0, \\
& \lambda^{g} \geq 0, \lambda_{i}^{g}=0, \forall i \notin I_{g}, \lambda_{i}^{\phi}=0, \forall i \notin I_{\phi}, \lambda_{i}^{\psi}=0, \forall i \notin I_{\psi}, \\
& \text { either } \lambda_{i}^{\phi}<0, \lambda_{i}^{\psi}<0 \text { or } \lambda_{i}^{\phi} \lambda_{i}^{\psi}=0 \quad \forall i \in I_{\phi} \cap I_{\psi} \\
& \Longrightarrow\left(\lambda^{g}, \lambda^{h}, \lambda^{\phi}, \lambda^{\psi}\right)=0 .
\end{aligned}
$$

(iv) MPEC quasi-normality holds at $z^{*}$ :

$$
\begin{aligned}
& \left\{\begin{array}{l}
(22)-(24) \text { and there exists a sequence }\left\{z^{k}\right\} \text { converging to } z^{*} \text { such that for each } k, \\
\lambda_{i}^{g}>0 \Rightarrow \lambda_{i}^{g} g_{i}\left(z^{k}\right)>0, \lambda_{i}^{h} \neq 0 \Rightarrow \lambda_{i}^{h} h_{i}\left(z^{k}\right)>0, \\
\lambda_{i}^{\phi} \neq 0 \Rightarrow \lambda_{i}^{\phi} \phi_{i}\left(z^{k}\right)<0, \lambda_{i}^{\psi} \neq 0 \Rightarrow \lambda_{i}^{\psi} \psi_{i}\left(z^{k}\right)<0
\end{array}\right. \\
& \Longrightarrow\left(\lambda^{g}, \lambda^{h}, \lambda^{\phi}, \lambda^{\psi}\right)=0 .
\end{aligned}
$$

(v) There is no nonzero direction in the MPEC linearized cone: $\mathcal{L}^{M P E C}\left(z^{*}\right)=\{0\}$ where

$$
\mathcal{L}^{M P E C}\left(z^{*}\right):=\left\{\begin{array}{l|l}
\nabla g_{i}\left(z^{*}\right) w \leq 0, i \in I_{g}, \nabla h_{i}\left(z^{*}\right) w=0, i=1, \ldots, q \\
\nabla \phi_{i}\left(z^{*}\right) w=0, i \in I_{\psi}^{c}, \nabla \psi_{i}\left(z^{*}\right) w=0, i \in I_{\phi}^{c} \\
0 \leq \nabla \phi_{i}\left(z^{*}\right) w \perp \nabla \psi_{i}\left(z^{*}\right) w \geq 0 \quad i \in I_{\phi} \cap I_{\psi}
\end{array}\right\} .
$$


(vi) The set-valued mapping $M_{1}(z):=(g(z), h(z))-\mathbb{R}_{-}^{p} \times\{0\}^{q}$ is metrically subregular at $\left(z^{*}, 0\right)$ and for every $0 \neq w \in \mathcal{L}^{M P E C}\left(z^{*}\right)$, one has

$$
\begin{aligned}
& \text { (22) }-(\underline{23}) \text { and }\left(\lambda_{i}^{\phi}, \lambda_{i}^{\psi}\right) \in N_{\Theta}\left(\nabla \phi_{i}\left(z^{*}\right) w, \nabla \psi_{i}\left(z^{*}\right) w\right) \forall i \in I_{\phi} \cap I_{\psi} \\
& \Longrightarrow\left(\lambda^{\phi}, \lambda^{\psi}\right)=0,
\end{aligned}
$$

where $N_{\Theta}(x, y)$ is given in (26).

(vii) The set-valued mapping $M_{1}(z):=(g(z), h(z))-\mathbb{R}_{-}^{p} \times\{0\}^{q}$ is metrically subregular at $\left(z^{*}, 0\right)$ and for every $0 \neq w \in \mathcal{L}^{M P E C}\left(z^{*}\right)$, one has

$$
\text { (25) and } w^{T} \nabla_{z}^{2} L^{0}\left(z^{*}, \lambda^{g}, \lambda^{h}, \lambda^{\phi}, \lambda^{\psi}\right) w \geq 0 \Longrightarrow\left(\lambda^{\phi}, \lambda^{\psi}\right)=0 \text {, }
$$

where

$$
L^{0}\left(z, \lambda^{g}, \lambda^{h}, \lambda^{\phi}, \lambda^{\psi}\right):=g(z)^{T} \lambda^{g}+h(z)^{T} \lambda^{h}+\phi(z)^{T} \lambda^{\phi}+\psi(z)^{T} \lambda^{\psi} .
$$

Proof. (i) follows from the corollary in [29, page 210]. (ii) is stronger than (iii), which is further stronger than (iv). (iv) follows from [17, Theorem 5.2]. (v) is the trivial case of (vi). (vi) and (vii) follow from [14, Theorem 2.6] and the well-known fact that the limiting normal cone of the complementarity cone $\Theta$ is equal to

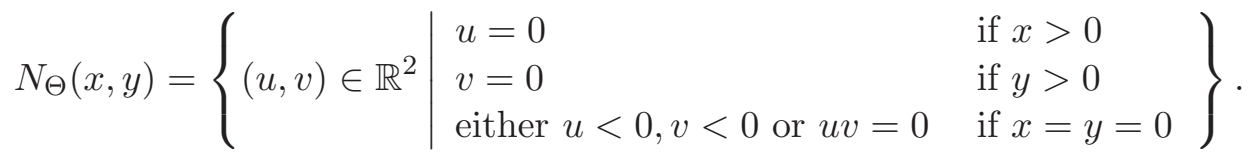

We now consider the following SOCCP

$$
\begin{aligned}
& \mathcal{K} \ni G(z) \perp H(z) \in \mathcal{K}, \\
& g(z) \leq 0, h(z)=0,0 \leq \phi(z) \perp \psi(z) \geq 0,
\end{aligned}
$$

where the second-order cone complementarity system (27) is defined as in (11) and $g: \mathbb{R}^{n} \rightarrow$ $\mathbb{R}^{p}, h: \mathbb{R}^{n} \rightarrow \mathbb{R}^{q}, \phi: \mathbb{R}^{n} \rightarrow \mathbb{R}^{s}, \psi: \mathbb{R}^{n} \rightarrow \mathbb{R}^{s}, G: \mathbb{R}^{n} \rightarrow \mathbb{R}^{m}, H: \mathbb{R}^{n} \rightarrow \mathbb{R}^{m}$ are continuously differentiable. Let the linearized cone of the system (27)-(28) be

$$
\mathcal{L}\left(z^{*}\right):=\left\{w \mid \begin{array}{l}
\nabla g_{i}\left(z^{*}\right) w \leq 0, i \in I_{g}, \nabla h_{i}\left(z^{*}\right) w=0, i=1, \ldots, q \\
\nabla \phi_{i}\left(z^{*}\right) w=0, i \in I_{\psi}^{c}, \nabla \psi_{i}\left(z^{*}\right) w=0, i \in I_{\phi}^{c} \\
0 \leq \nabla \phi_{i}\left(z^{*}\right) w \perp \nabla \psi_{i}\left(z^{*}\right) w \geq 0 \quad i \in I_{\phi} \cap I_{\psi} \\
\left(\nabla G_{i}\left(z^{*}\right) w, \nabla H_{i}\left(z^{*}\right) w\right) \in T_{\Omega_{i}}\left(G_{i}\left(z^{*}\right), H_{i}\left(z^{*}\right)\right), i=1, \ldots, J
\end{array}\right\},
$$

where $T_{\Omega_{i}}$ can be calculated as in Theorem 5.1. Based on the results we obtained, we now derive a sufficient condition for error bounds for the system (27)-(28) that are explicitly verifiable based on the initial data.

Theorem 7.6 Given a point $z^{*} \in \mathcal{F}$. Suppose that the complementarity system (28) is metrically subregular at $\left(z^{*}, 0\right)$. Further assume that either $\mathcal{L}\left(z^{*}\right)=\{0\}$, or $\mathcal{L}\left(z^{*}\right) \neq\{0\}$ 
and for every $0 \neq w \in \mathcal{L}\left(z^{*}\right)$ one has

$$
\begin{aligned}
& \left\{\begin{array}{l}
\nabla g\left(z^{*}\right)^{T} \lambda^{g}+\nabla h\left(z^{*}\right)^{T} \lambda^{h}+\nabla \phi\left(z^{*}\right)^{T} \lambda^{\phi}+\nabla \psi\left(z^{*}\right)^{T} \lambda^{\psi}+\nabla G\left(z^{*}\right)^{T} \lambda^{G}+\nabla H\left(z^{*}\right)^{T} \lambda^{H}=0, \\
\lambda^{g} \geq 0, \lambda_{i}^{g}=0 \forall i \notin I_{g}, \lambda_{i}^{\phi}=0 \forall i \notin I_{\phi}, \lambda_{i}^{\psi}=0 \forall i \notin I_{\psi}, \\
\left(\lambda_{i}^{\phi}, \lambda_{i}^{\psi}\right) \in N_{\Theta}\left(\nabla \phi_{i}\left(z^{*}\right) w, \nabla \psi_{i}\left(z^{*}\right) w\right) \forall i \in I_{\phi} \cap I_{\psi}, \\
\left(\lambda_{i}^{G}, \lambda_{i}^{H}\right) \in N_{\Omega_{i}}\left(\left(G_{i}\left(z^{*}\right), H_{i}\left(z^{*}\right)\right) ;\left(\nabla G_{i}\left(z^{*}\right) w, \nabla H_{i}\left(z^{*}\right) w\right)\right), \forall i=1, \ldots, J,
\end{array}\right. \\
& \Longrightarrow\left(\lambda^{G}, \lambda^{H}\right)=0,
\end{aligned}
$$

where $N_{\Theta}(\cdot)$ is given in (26) and $N_{\Omega_{i}}((x, y) ;(d, w))$ can be calculated as in Theorem 6.1. Then the system (27)- (28) has a local error bound at $z^{*}$. That is, there exist a constant $\kappa>0$ and $\delta>0$ such that

$d(z, \mathcal{F}) \leq \kappa\left\{\|h(z)\|+\left\|g_{+}(z)\right\|+\sum_{i=1}^{s} d_{\Theta}\left(\phi_{i}(z), \psi_{i}(z)\right)+\sum_{i=1}^{J} d_{\Omega_{i}}\left(G_{i}(z), H_{i}(z)\right)\right\}, \forall z \in B_{\delta}\left(z^{*}\right)$.

Proof. To prove the result, we take $F(z):=(g(z), h(z), \phi(z), \psi(z))$ and $\Lambda:=\mathbb{R}_{-}^{p} \times\{0\}^{q} \times \Theta^{s}$ and apply Theorem 7.3 . Since the sets $\mathbb{R}_{-}^{p},\{0\}^{q}$ are convex and $\Theta^{s}$ is directionally regular, we have

$$
\begin{aligned}
& T_{\Lambda}\left(F\left(z^{*}\right)\right)=T_{\mathbb{R}_{-}^{p}}\left(g\left(z^{*}\right)\right) \times T_{\{0\}^{q}}\left(h\left(z^{*}\right)\right) \times T_{\Theta^{s}}\left(\phi\left(z^{*}\right), \psi\left(z^{*}\right)\right), \\
& N_{\Lambda}\left(F\left(z^{*}\right) ; \nabla F\left(z^{*}\right) w\right) \\
& =N_{\mathbb{R}_{-}^{p}}\left(g\left(z^{*}\right) ; \nabla g\left(z^{*}\right) w\right) \times N_{\{0\}^{q}}\left(h\left(z^{*}\right) ; \nabla h\left(z^{*}\right) w\right) \times N_{\Theta^{s}}\left(\left(\phi\left(z^{*}\right), \psi\left(z^{*}\right)\right) ;\left(\nabla \phi\left(z^{*}\right) w, \nabla \phi\left(z^{*}\right) w\right)\right), \\
& N_{\Theta^{s}}\left(\left(\phi\left(z^{*}\right), \psi\left(z^{*}\right)\right) ;\left(\nabla \phi\left(z^{*}\right) w, \nabla \phi\left(z^{*}\right) w\right)\right)=\Pi_{i=1}^{s} N_{\Theta}\left(\left(\phi_{i}\left(z^{*}\right), \psi_{i}\left(z^{*}\right)\right) ;\left(\nabla \phi_{i}\left(z^{*}\right) w, \nabla \phi_{i}\left(z^{*}\right) w\right)\right) .
\end{aligned}
$$

Moreover by Proposition 3.4 and Theorem 6.1, for all $w$ such that $\left(\nabla \phi_{i}\left(z^{*}\right) w, \nabla \psi_{i}\left(z^{*}\right) w\right) \in$ $T_{\Theta}\left(\phi_{i}\left(z^{*}\right), \psi_{i}\left(z^{*}\right)\right)$, we have

$$
\begin{aligned}
& N_{\Theta}\left(\left(\phi_{i}\left(z^{*}\right), \psi_{i}\left(z^{*}\right)\right) ;\left(\nabla \phi_{i}\left(z^{*}\right) w, \nabla \phi_{i}\left(z^{*}\right) w\right)\right) \\
& \quad= \begin{cases}\mathbb{R} \times\{0\} & \text { if } \phi_{i}\left(z^{*}\right)=0, \psi_{i}\left(z^{*}\right)>0, \\
\{0\} \times \mathbb{R} & \text { if } \phi_{i}\left(z^{*}\right)>0, \psi_{i}\left(z^{*}\right)=0, \\
N_{\Theta}\left(\nabla \phi_{i}\left(z^{*}\right) w, \nabla \psi_{i}\left(z^{*}\right) w\right) & \text { if } \phi_{i}\left(z^{*}\right)=0, \psi_{i}\left(z^{*}\right)=0 .\end{cases}
\end{aligned}
$$

By using the tangent cone formula in Corollary 5.1 and the limiting normal cone formula in (26), we derive the result.

Acknowledgments. The authors are grateful to the two anonymous referees for their helpful comments and suggestions.

\section{References}

[1] F. Alizadeh and D. Goldfarb, Second-order cone programming, Math. Program., 95(2003), pp. 3-51.

[2] J.-P. Aubin, Lipschitz behavior of solutions to convex minimization problems, Math. Oper. Res., 9(1984), pp. 87-111. 
[3] J.F. Bonnans and H. RamŔez C., Perturbation analysis of second-order cone programming problems, Math. Program., 104(2005), pp. 205-227.

[4] J.-S. Chen, X. Chen And P. Tseng, Analysis of nonsmooth vector-valued functions associated with second-order cone, Math. Program., 101(2004), pp. 95-117.

[5] J.-S. Chen And P. Tseng, An unconstrained smooth minimization reformulation of the second-order cone complementarity problem, Math. Program., 104(2005), pp. 293-327.

[6] F.H. Clarke, Optimization and Nonsmooth Analysis, Wiley-Interscience, New York, 1983.

[7] F.H. Clarke, Yu. S. Ledyaev, R.J. Stern and P.R. Wolenski, Nonsmooth Analysis and Control Theory, Springer, New York, 1998.

[8] A.L. Dontchev and R.T. Rockafellar, Implicit Functions and Solution Mappings, Springer, Heidelberg, 2014.

[9] M.J. Fabian, R. Henrion, A.Y. Kruger and J.V. Outrata, Error bounds: necessary and sufficient conditions, Set-Valued Var. Anal., 18(2010), pp. 121-149.

[10] F. Facchinei, A. Fischer And M. Herrich, An LP-Newton method: nonsmooth equations, KKT systems, and nonisolated solutions, Math. Program., 146(2014), pp. $1-36$.

[11] M.L. Flegel, C. Kanzow and J.V. Outrata, Optimality conditions for disjunctive programs with application to mathematical programs with equilibrium constraints, SetValued Anal., 15(2007), pp. 139-162.

[12] H. GFrener, First order and second order characterizations of metric subregularity and calmness of constraint set mappings, SIAM J. Optim., 21(2011), pp. 1439-1474.

[13] H. GFrerer, On directional metric regularity, subregularity and optimality conditions for nonsmooth mathematical programs, Set-Valued Var. Anal., 21(2013), pp. 151-176.

[14] H. GFrerer, Optimality conditions for disjunctive programs based on generalized differentiation with application to mathematical programs with equilibrium constraints, SIAM J. Optim., 24(2014), pp. 898-931.

[15] H. Gfrerer, D. Klatte, Lipschitz and Hölder stability of optimization problems and generalized equations, Math. Program., 158(2016), pp. 35-75.

[16] H. GFrerer And J.J. Ye, New constraint qualifications for mathematical programs with equilibrium constraints via variational analysis, SIAM J. Optim., 27(2017), pp. 842-865.

[17] L. Guo, J.J. Ye And J. ZhAng, Mathematical programs with geometric constraints in Banach spaces: enhanced optimality, exact penalty, and sensitivity, SIAM J. Optim., 23(2013), pp. 2295-2319.

[18] L. Guo, J. Zhang And G.-H. Lin, New results on constraint qualifications for nonlinear extremum problems and extensions, J. Optim. Theory Appl., 163(2014), pp. 737-754. 
[19] S. Hayashi, N. Yamashita And M. Fukushima, Robust Nash equilibria and secondorder cone complementarity problems, J. Nonl. Conv. Anal., 6(2005), pp. 283-296.

[20] Y. JiAng, Y.J. LiU AND L.W. Zhang, Variational geometry of the complementarity set for second order cone, Set-Valued Var. Anal., 23(2015), pp. 399-414.

[21] D. Klatte And B. Kummer, Nonsmooth Equations in Optimization: Regularity, Calculus, Methods and Applications, Kluwer Academic Publishers, 2002.

[22] M.S. Lobo, L. Vandenberghe, S. Boyd and H. Lebret, Applications of secondorder cone programming, Linear Algebra Appl., 284(1998), pp. 193-228.

[23] B.S. Mordukhovich, Variational Analysis and Generalized Differentiation, Vol. I: Basic Theory, Vol. II: Applications, Springer, Berlin, 2006.

[24] K.F. NG And X.Y. Zheng, Global error bounds with fractional exponents, Math. Program., 88(2000), pp. 357-370.

[25] J.V. Outrata And D.F. Sun, On the coderivative of the projection operator onto the second-order cone, Set-Valued Anal., 16(2008), pp. 999-1014.

[26] J.S. PANG, Error bounds in mathematical programming, Math. Program., 79(1997), pp. 299-332.

[27] S.M. Robinson, Stability theory for systems of inequality constraints, part I: linear systems, SIAM J. Numer. Anal., 12(1975), pp. 754-769.

[28] S.M. Robinson, Stability theory for systems of inequality constraints, part II: differentiable nonlinear systems, SIAM J. Numer. Anal., 13(1976), pp. 497-513.

[29] S.M. Robinson, Some continuity properties of polyhedral multifunctions, Math. Program. Stud., 14(1981), pp. 206-214.

[30] R.T. Rockafellar and R. J-B. Wets, Variational Analysis, Springer, Berlin, 1998.

[31] Z.L. Wu AND J.J. YE, Sufficient conditions for error bounds, SIAM J. Optim., 12(2001), pp. 421-435.

[32] Z.L. Wu AND J.J. YE, On error bounds for lower semicontinuous functions, Math. Program., 92(2002), pp. 301-314.

[33] Z.L. Wu AND J.J. YE, First-order and second-order conditions for error bounds, SIAM J. Optim., 14(2003), pp. 621-645.

[34] J.J. YE, Constraint qualification and necessary optimality conditions for optimization problems with variational inequality constraints, SIAM J. Optim., 10(2000), pp. 943962.

[35] J.J. YE AND X.Y. YE, Necessary optimality conditions for optimization problems with variational inequality constraints, Math. Oper. Res., 22(1997), pp. 977-997.

[36] J.J. YE AND J.C. ZHOU, First-order optimality conditions for mathematical programs with second-order cone complementarity constraints, SIAM J. Optim., 26(2016), pp. 2820-2846. 
[37] J.J. Ye AND J.C. ZHou, Exact formula for the proximal/regular/limiting normal cone of the second-order cone complementarity set, Math. Program., 162(2017), pp. 33-50. 